\title{
A variational approach to resistive relativistic plasmas
}

\author{
N. Andersson ${ }^{1}$, G.L. Comer ${ }^{2}$ and I.Hawke ${ }^{1}$ \\ 1 Mathematical Sciences and STAG Research Centre, \\ University of Southampton, Southampton SO17 1BJ, United Kingdom \\ 2 Department of Physics, Saint Louis University, St. Louis, MO, 63156-0907, USA
}

(Dated: April 4, 2017)

\begin{abstract}
We develop an action principle to construct the field equations for a multi-fluid system containing charge-neutral fluids, plasmas, and dissipation (via resistive interactions), by combining the standard, Maxwell action and minimal coupling of the electromagnetic field with a recently developed action for relativistic dissipative fluids. We use a pull-back formalism from spacetime to abstract matter spaces to build unconstrained variations for both the charge-neutral fluids and currents making up the plasmas. Using basic linear algebra techniques, we show that a general "relabeling" invariance exists for the abstract matter spaces. With the field equations in place, a phenomenological model for the resistivity is developed, using as constraints charge conservation and the Second Law of Thermodynamics. A minimal model for a system of electrons, protons, and heat is developed using the Onsager procedure for incorporating dissipation.
\end{abstract}

PACS numbers: 04.40.Nr, 04.40.-b, 03.50.-z 


\section{INTRODUCTION}

Relativistic fluid dynamics is a well developed area of research, with exciting applications ranging from astrophysics to high-energy collider physics (see Andersson and Comer [1]). These applications become more complex and involved as our computational technology advances. In astrophysics, the state-of-the-art is represented by neutron-star simulations (or work on supernova core collapse) including multi-dimensional neutrino transport $[2,3]$ and compact mergers of magnetised binary stars including resistive effects $[4,5]$. Meanwhile, the high-energy physics problem has inspired the first simulations of second-order, causal, dissipative models, building on the classic formulation of Israel and Stewart [6, 7]. In parallel, there have been formal developments of the theory (including many relevant efforts in the string-theory inspired area of holography, see for example, [8]).

When it comes to classical (general-) relativistic fluid dynamics, the most interesting developments involve the consideration of multi-fluid systems, e.g. issues arising when components become superfluid, when heat flows and when the electromagnetic charge current is treated as a dynamical variable [1,9-11]. These advances allow us to consider a wide range of relevant phenomena, but the general theory is incomplete in two important respects. First of all, we need to be able to consider dissipation (for which a plethora of mechanisms may operate in a multifluid system). Secondly, we need to couple the dissipative fluid dynamics to electromagnetism. The former poses a formal challenge because, while it is well-known that non-dissipative fluid dynamics can be derived from an action principle [1, 12-15], the inclusion of dissipation in these systems tends to be phenomenological. The second is key if we want to move towards a greater level of realism in our astrophysics modeling.

Given the first of these two issues, the recently proposed strategy for extending the variational approach to dissipative systems [16] is promising. In principle, it provides us with an avenue for connecting dissipative channels with the underlying matter description and equation of state models accounting for transport phenomena. This paper aims to address the second issue by extending the variational derivation to account for electromagnetism. In particular, we provide a variational derivation for charged multifluid systems, accounting for particle reactions and resistive scattering. Having obtained the formal results we discuss issues relating to electromagnetic gauge-invariance and develop a phenomenological model inspired by (and consistent with) the formal results. These developments provide a robust 
foundation for applications, as discussed in two companion papers [17, 18].

In Sec. II, we discuss the fundamental variables of the system and review the pull-back formalism. We also show how to build in general re-labeling invariance for the matter spaces. The total action, its variation, and resulting field equations are given in Sec. III. In Sec. IV, we use a decomposition of the system variables and fluid field equations with respect to a local "observer's" frame-of-reference to illuminate various features of the resistivity and to

exploit them so as to produce a phenomenological model. A minimal model for a system of electrons, protons, and heat is provided in Sec. V. Concluding thoughts and some discussion of immediate applications of the formalism are presented in Sec. VI. Finally, in an appendix, we show how minimal coupling can be considered as a special type of entrainment between the electromagnetic four-potential and the charged fluid fluxes. The conventions of Misner, Thorne, and Wheeler [19] are used throughout.

\section{SYSTEM KINEMATICS: THE FIELDS AND VARIABLES}

We will assume that our system has a number $N_{c}$ of independent fluid constituents (such as electrons, protons, neutrons, and entropy). Each constituent has as its fundamental field a particle number density current $n_{\mathrm{x}}^{a}$, where $\mathrm{x}$ is a label that ranges over the various $N_{c}$ constituents (e for electrons, $\mathrm{p}$ for protons, etc.). The density $n_{\mathrm{x}}$ associated with a given flux is given by $n_{\mathrm{x}}^{2}=-g_{a b} n_{\mathrm{x}}^{a} n_{\mathrm{x}}^{b}$. Among the $N_{c}$ constituents there will be a number $N_{q}$ which are charged, such that $N_{q} \leq N_{c}$. Each of these will have a charge $e_{\mathrm{x}}$ which combines with its associated flux current $n_{\mathrm{x}}^{a}$ to give a charged flux current $j_{\mathrm{x}}^{a}=e_{\mathrm{x}} n_{\mathrm{x}}^{a}$. Associated with each flux is a (canonically conjugate) fluid momentum $\mu_{a}^{\mathrm{x}}[c f$. Eq. (58)]. While not dynamically independent (being a function of, in principle, all of the fluxes), its identification is an important step towards extracting various physical properties of the system - such as vorticity [cf. Eq. (59)]. The remaining field variables are the four-vector potential $A_{a}$ and the spacetime metric $g_{a b}$. With $A_{a}$ we couple the charged fluids to the electromagnetic field (and vice versa); the metric couples all fields to the spacetime curvature (and vice versa). At the end we have a complete system for describing a system of charged, self-gravitating, relativistic fluids. 


\section{A. Fluid Particle Worldlines and Fluid Matter Space}

The (charged and uncharged) "fluid particles" associated with a given flux will have worldlines that follow from the unit four-velocity field $u_{\mathrm{x}}^{a}=n_{\mathrm{x}}^{a} / n_{\mathrm{x}}$. In general, the number of independent four-velocities, or equivalently, the number of (charged and uncharged) fluids, $N_{f}$ will be equal to or less than $N_{c}$. This is determined from the outset by the details of the system that is to be described. When $N_{f}=N_{c}$, each constituent can move independently of the others, but when $N_{f}<N_{c}$, some of the constituents are flowing together; for example, as the limit of dynamical locking due to the resistive form of interaction developed later.

In Fig. 1 we have a representation of some fluid-element worldlines, for a system of two fluids. With respect to the local coordinate system $\left\{x^{0}, x^{i}\right\}$, the points on the left-most, $\mathrm{x}$-fluid worldline are given by $x_{\mathrm{x}}^{a}(\tau)$, where $\tau$ is the proper time. The functions $x_{\mathrm{x}}^{a}(\tau)$ can be constructed from $\mathrm{d} x_{\mathrm{x}}^{a} / \mathrm{d} \tau=u_{\mathrm{x}}^{a}$ once the fluid field equations are satisfied and $u_{\mathrm{x}}^{a}$ is known. Likewise, for the right-most, $\mathrm{y}$-fluid worldline, the functions $x_{\mathrm{y}}^{a}(\lambda)$, where $\lambda$ is the proper time, come from integrating $\mathrm{d} x_{\mathrm{y}}^{a} / \mathrm{d} \lambda=u_{\mathrm{y}}^{a}$, once the $u_{\mathrm{y}}^{a}$ are known.

With respect to system evolution, one often has in mind an initial-value approach to finding solutions. In our local coordinate system, we have an initial, spacelike slice at $x^{0}=0$, and so our determination of $x_{\mathrm{x}}^{a}(\tau)$ and $x_{\mathrm{y}}^{a}(\lambda)$ for the two worldlines will be based on the specification of their respective initial locations, namely, $x_{\mathrm{x}}^{a}\left(\tau_{0}\right)$ and $x_{\mathrm{y}}^{a}\left(\lambda_{0}\right)$. This brings up an important point: Assuming a given initial slice, and the fact that proper time allows for some re-parametrization invariance, we see that $x_{\mathrm{x}}^{i}\left(\tau_{0}\right)$ for each worldline is all that is needed to set up the distribution of the worldlines on the initial slice. In fact, it is

plausible that once this labeling is in place, each fluid element will carry along with itself (via Lie-dragging) its original label as it moves along its worldline.

This leads us to introduce for each fluid an abstract, three-dimensional "matter" space, the coordinates of which can be used as dynamical variables for the fluids $[12,13]$. The role of the equations of motion is to guarantee that the initial set-up will lead to families of worldlines as fibrations of spacetime. On the left in Fig. 1 we have placed the x-fluid matter space having coordinates $X_{\mathrm{x}}^{A}, A=\{\mathbf{1}, \mathbf{2}, \mathbf{3}\}$, and on the right is the $\mathrm{y}$-fluid matter space with coordinates $X_{\mathrm{y}}^{A}$. As we see in the figure, a "point" in either matter space is identified with a worldline of a fluid element in spacetime. The $X_{\mathrm{x}}^{A}$ are in general a set of three scalars on spacetime. They only vary from worldline to worldline, meaning for all $\tau$ 


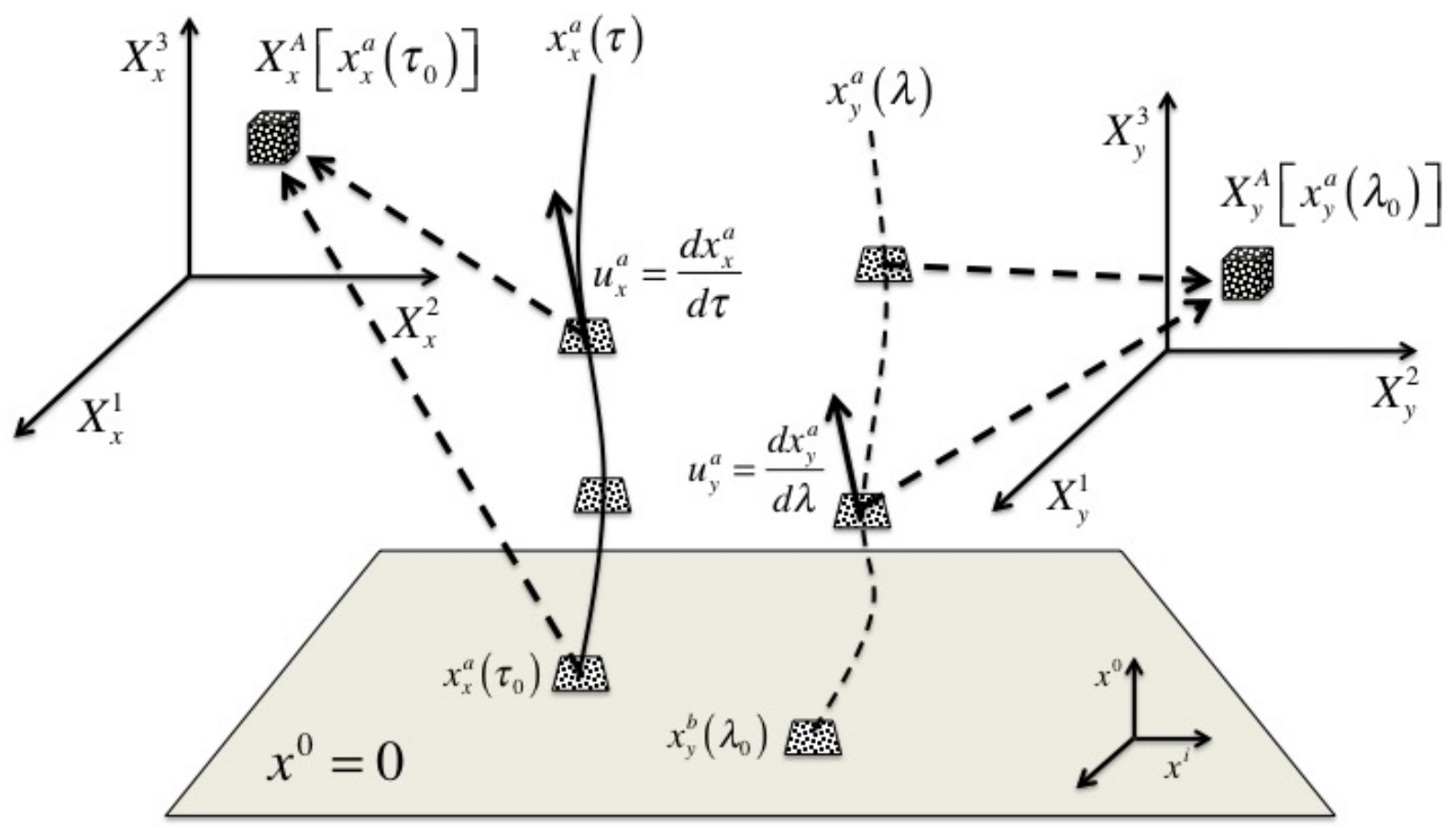

FIG. 1: A representation of the pull-back description for fluids based on matching worldlines in spacetime to points in matter space. We have placed on the worldlines small squares filled with dots. This is to emphasize the fact that the worldlines are for fluid elements, and not individual particles, and thus "points" on the worldlines are best thought of as small (with respect to the whole system) boxes containing a (thermodynamically describable) large number of particles.

of each worldline (and $\lambda$ for the other fluid worldlines)

$$
X_{\mathrm{x}}^{A}\left[\mathrm{x}_{\mathrm{x}}^{a}(\tau)\right]=X_{\mathrm{x}}^{A}\left[0, x_{\mathrm{x}}^{i}\left(\tau_{0}\right)\right],
$$

yet, for two different worldlines at, say, $\left\{0, x_{\mathrm{x}}^{i}\left(\tau_{0}\right)\right\}$ and $\left\{0, x_{\mathrm{x}}^{i}\left(\tau_{0}\right)+\delta x^{i}\right\}$, we have

$$
X_{\mathrm{x}}^{A}\left[0, x_{\mathrm{x}}^{i}\left(\tau_{0}\right)\right] \neq X_{\mathrm{x}}^{A}\left[0, x_{\mathrm{x}}^{i}\left(\tau_{0}\right)+\delta x^{i}\right] .
$$

Next we will show how the $X_{\mathrm{x}}^{A}$ can be used as the fundamental fields for modeling fluid dynamics.

\section{B. Pull-back Formalism}

Consider the three-form $n_{a b c}^{\mathrm{x}}$ which is dual to $n_{\mathrm{x}}^{a}$ :

$$
n_{a b c}^{\mathrm{x}}=\epsilon_{d a b c} n_{\mathrm{x}}^{d} \quad, \quad n_{\mathrm{x}}^{a}=\frac{1}{3 !} \epsilon^{b c d a} n_{b c d}^{\mathrm{x}},
$$


where our convention for transforming between the two is

$$
\epsilon^{b c d a} \epsilon_{e b c d}=3 ! \delta_{e}^{a}
$$

Likewise, we introduce

$$
\mu_{\mathrm{x}}^{a b c}=\epsilon^{d a b c} \mu_{d}^{\mathrm{x}} \quad, \quad \mu_{a}^{\mathrm{x}}=\frac{1}{3 !} \epsilon_{b c d a} \mu_{\mathrm{x}}^{b c d}
$$

which is the three-form dual to $\mu_{a}^{\mathrm{x}}$.

We use the $X_{\mathrm{x}}^{A}$ to "pullback" $n_{a b c}^{\mathrm{x}}$ into the matter space where it is identified with $n_{A B C}^{\mathrm{x}}$ :

$$
n_{a b c}^{\mathrm{x}}={ }^{\mathrm{x}} \mathcal{J}_{a b c}^{A B C} n_{A B C}^{\mathrm{x}},
$$

such that the Einstein convention applies to repeated matter space indices, and

$$
\times \mathcal{J}_{a b c}^{A B C}=\frac{\partial X_{\mathrm{x}}^{[A}}{\partial x^{a}} \frac{\partial X_{\mathrm{x}}^{B}}{\partial x^{b}} \frac{\partial X_{\mathrm{x}}^{C]}}{\partial x^{c}} .
$$

We also use the $X_{\mathrm{x}}^{A}$ to "push-forward" a matter space quantity, $\mu_{\mathrm{x}}^{A B C}$, to the spacetime three-form $\mu_{\mathrm{x}}^{a b c}$ :

$$
\mu_{\mathrm{x}}^{A B C}={ }^{\mathrm{x}} \mathcal{J}_{a b c}^{A B C} \mu_{\mathrm{x}}^{a b c} .
$$

Note that this construction leads to $X_{\mathrm{x}}^{A}$ which are conserved along their own worldlines (i.e. they are Lie-dragged by their $u_{\mathrm{x}}^{a}$ ):

$$
\frac{\mathrm{d} X_{\mathrm{x}}^{A}}{\mathrm{~d} \tau_{\mathrm{x}}}=u_{\mathrm{x}}^{a} \nabla_{a} X_{\mathrm{x}}^{A}=\frac{1}{n_{\mathrm{x}}}\left(-\frac{1}{3 !} \epsilon^{a b c d} \frac{\partial X_{\mathrm{x}}^{A}}{\partial x^{[a}} \frac{\partial X_{\mathrm{x}}^{B}}{\partial x^{b}} \frac{\partial X_{\mathrm{x}}^{C}}{\partial x^{c}} \frac{\partial X_{\mathrm{x}}^{D}}{\partial x^{d]}}\right) n_{B C D}^{\mathrm{x}} \equiv 0
$$

since the term in parentheses vanishes identically.

Because of the antisymmetry in its indices, $n_{A B C}^{\mathrm{x}}$ allows a natural definition for a volumeform $\epsilon_{A B C}^{\mathrm{x}}$ - up to a normalization convention to be established in the next subsection on the x-matter space:

$$
n_{A B C}^{\mathrm{x}}=\mathcal{N}^{\mathrm{x}} \epsilon_{A B C}^{\mathrm{x}},
$$

where $\mathcal{N}^{\mathrm{x}}$ will be defined momentarily. Similarly, the antisymmetry of the indices of $\mu_{\mathrm{x}}^{A B C}$ leads to an "inverse" volume form; namely,

$$
\mu_{\mathrm{x}}^{A B C}=\mathcal{M}_{\mathrm{x}} \epsilon_{\mathrm{x}}^{A B C}
$$

where $\mathcal{M}_{\mathrm{x}}$ will also be defined momentarily. The quantity $\epsilon_{\mathrm{x}}^{A B C}$ is inverse in the sense that we impose

$$
\epsilon_{D E F}^{\mathrm{x}} \epsilon_{\mathrm{x}}^{A B C}=3 ! \delta_{D}^{[A} \delta_{E}^{B} \delta_{F}^{C]}
$$


which implies

$$
\epsilon_{A B C}^{\mathrm{x}} \epsilon_{\mathrm{x}}^{A B C}=3 !
$$

Now, we can write

$$
\mathcal{N}^{\mathrm{x}}=\frac{1}{3 !} \epsilon_{\mathrm{x}}^{A B C} n_{A B C}^{\mathrm{x}} \quad, \quad \mathcal{M}_{\mathrm{x}}=\frac{1}{3 !} \epsilon_{A B C}^{\mathrm{x}} \mu_{\mathrm{x}}^{A B C} .
$$

Letting

$$
n_{\mathrm{x}}=-n_{\mathrm{x}}^{a} u_{a}^{\mathrm{x}} \quad, \quad \mu^{\mathrm{x}}=-u_{\mathrm{x}}^{a} \mu_{a}^{\mathrm{x}}
$$

we have

$$
\mu^{\mathrm{x}} n_{\mathrm{x}}=\mathcal{M}_{\mathrm{x}} \mathcal{N}^{\mathrm{x}}
$$

\section{Matter Space Metrics}

To complete the establishment of $\epsilon_{A B C}^{\mathrm{x}}$ as a volume measure on its associated matter space, we need to establish normalizations for it and $\epsilon_{\mathrm{x}}^{A B C}$. Because of their antisymmetry, $\epsilon_{A B C}^{\mathrm{x}}$ and $\epsilon_{\mathrm{x}}^{A B C}$ each have only one independent component: $\epsilon_{123}^{\mathrm{x}}$ and $\epsilon_{\mathrm{x}}^{123}$, respectively. Establishing a normalization for $\epsilon_{A B C}^{\mathrm{x}}$ and $\epsilon_{\mathrm{x}}^{A B C}$ means setting values for $\epsilon_{123}^{\mathrm{x}}$ and $\epsilon_{\mathrm{x}}^{123}$. We will use a standard, linear algebra approach (see, Strang [20]) which, among other things, leads to re-labeling invariance for the matter spaces.

Note that the particle number densities can now take the form

$$
n_{\mathrm{x}}^{2}=\left(\sqrt{g_{\mathrm{x}}} \mathcal{N}^{\mathrm{x}}\right)^{2}
$$

where

$$
g_{\mathrm{x}}=\frac{1}{3 !} \epsilon_{A B C}^{\mathrm{x}} \epsilon_{D E F}^{\mathrm{x}} g_{\mathrm{x}}^{A D} g_{\mathrm{x}}^{B E} g_{\mathrm{x}}^{C F} \quad, \quad g_{\mathrm{x}}^{A B}=\frac{\partial X_{\mathrm{x}}^{A}}{\partial x^{a}} \frac{\partial X_{\mathrm{x}}^{B}}{\partial x^{b}} g^{a b}
$$

and

$$
n_{\mathrm{xy}}^{2}=g_{\mathrm{xy}} \mathcal{N}^{\mathrm{x}} \mathcal{N}^{\mathrm{y}}
$$

where

$$
g_{\mathrm{xy}}=\frac{1}{3 !} \epsilon_{A B C}^{\mathrm{x}} \epsilon_{D E F}^{\mathrm{y}} g_{\mathrm{xy}}^{A D} g_{\mathrm{xy}}^{B E} g_{\mathrm{xy}}^{C F} \quad, \quad g_{\mathrm{xy}}^{A B}=\frac{\partial X_{\mathrm{x}}^{A}}{\partial x^{a}} \frac{\partial X_{\mathrm{y}}^{B}}{\partial x^{b}} g^{a b} .
$$

We will use the determinants of $g_{\mathrm{x}}^{A B}$ and its inverse to form normalizations for $\epsilon_{A B C}^{\mathrm{x}}$ and $\epsilon_{\mathrm{x}}^{A B C}$.

The standard, matrix definition [20] for the determinant of $g_{\mathrm{x}}^{A B}$ is

$$
\Delta_{\mathrm{x}}=\frac{1}{3 !\left(\epsilon_{123}^{\mathrm{x}}\right)^{2}} \epsilon_{A B C}^{\mathrm{x}} \epsilon_{D E F}^{\mathrm{x}} g_{\mathrm{x}}^{A D} g_{\mathrm{x}}^{B E} g_{\mathrm{x}}^{C F} .
$$


The "matrix" inverse $g_{A B}^{\mathrm{x}}$ of $g_{\mathrm{x}}^{A B}$ is the solution to

$$
g_{\mathrm{x}}^{A C} g_{C B}^{\mathrm{x}}=g_{\mathrm{x}}^{C A} g_{C B}^{\mathrm{x}}=g_{B C}^{\mathrm{x}} g_{\mathrm{x}}^{C A}=g_{B C}^{\mathrm{x}} g_{\mathrm{x}}^{A C}=\delta_{B}^{A},
$$

and its determinant is

$$
\Delta^{\mathrm{x}}=\frac{1}{3 !\left(\epsilon_{\mathrm{x}}^{123}\right)^{2}} \epsilon_{\mathrm{x}}^{A B C} \epsilon_{\mathrm{x}}^{D E F} g_{A D}^{\mathrm{x}} g_{B E}^{\mathrm{x}} g_{C F}^{\mathrm{x}}
$$

Our last step is to impose

$$
\epsilon_{\mathrm{x}}^{A B C} \epsilon_{\mathrm{x}}^{D E F} g_{A D}^{\mathrm{x}} g_{B E}^{\mathrm{x}} g_{C F}^{\mathrm{x}}=\epsilon_{A B C}^{\mathrm{x}} \epsilon_{D E F}^{\mathrm{x}} g_{\mathrm{x}}^{A D} g_{\mathrm{x}}^{B E} g_{\mathrm{x}}^{C F}=3 !
$$

(which means $g_{\mathrm{x}}=1$ ) and thus find

$$
\epsilon_{123}^{\mathrm{x}}=\frac{1}{\epsilon_{\mathrm{x}}^{123}}=\sqrt{\Delta^{\mathrm{x}}}=\frac{1}{\sqrt{\Delta_{\mathrm{x}}}} .
$$

We will assume that the explicit form for $g_{A B}^{\mathrm{x}}$ must be a combination of $g_{\mathrm{x}}^{A B}$ and $\epsilon_{A B C}^{\mathrm{x}}$. Because $g_{\mathrm{x}}^{A B}$ is symmetric, and taking into account Eq. (12), the only combination is

$$
g_{A B}^{\mathrm{x}}=a_{\mathrm{x}} \epsilon_{A C E}^{\mathrm{x}} \epsilon_{B D F}^{\mathrm{x}} g_{\mathrm{x}}^{C D} g_{\mathrm{x}}^{E F} .
$$

To complete the solution, we note that

$$
g_{\mathrm{x}}^{A B} g_{A B}^{\mathrm{x}}=\delta_{A}^{A} \Longrightarrow a_{\mathrm{x}}=\frac{1}{2}
$$

It is straightforward to verify that

$$
g_{\mathrm{x}}^{A C}\left(\frac{1}{2} \epsilon_{C D F}^{\mathrm{x}} \epsilon_{B E G}^{\mathrm{x}} g_{\mathrm{x}}^{D E} g_{\mathrm{x}}^{F G}\right)=0 \quad, \quad A \neq B
$$

In a similar manner, we can find the inverse for $g_{\mathrm{xy}}^{A B}$. This is a bit trickier, as we are mixing coordinates of two different matter spaces. We will consider it to have a "left"- and a "right"-inverse:

$$
\begin{aligned}
& g_{B C}^{\mathrm{xy}} g_{\mathrm{yx}}^{C A}=g_{B C}^{\mathrm{xy}} g_{\mathrm{xy}}^{A C}=\delta_{B}^{A}, \\
& g_{\mathrm{xy}}^{A C} g_{C B}^{\mathrm{yx}}=g_{\mathrm{yx}}^{C A} g_{C B}^{\mathrm{yx}}=\delta_{B}^{A} .
\end{aligned}
$$

As before, we use $\epsilon_{\mathrm{x}}^{A B C}$ and $\epsilon_{A B C}^{\mathrm{x}}$ to calculate the determinants

$$
\begin{aligned}
& \Delta_{\mathrm{xy}}=\frac{1}{3 ! \epsilon_{123}^{\mathrm{x}} \epsilon_{123}^{\mathrm{y}}} \epsilon_{A B C}^{\mathrm{x}} \epsilon_{D E F}^{\mathrm{y}} g_{\mathrm{xy}}^{A D} g_{\mathrm{xy}}^{B E} g_{\mathrm{xy}}^{C F}=\Delta_{\mathrm{yx}} \\
& \Delta^{\mathrm{xy}}=\frac{1}{3 ! \epsilon_{\mathrm{x}}^{123} \epsilon_{\mathrm{y}}^{123}} \epsilon_{\mathrm{x}}^{A B C} \epsilon_{\mathrm{y}}^{D E F} g_{A D}^{\mathrm{xy}} g_{B E}^{\mathrm{xy}} g_{C F}^{\mathrm{xy}}=\Delta^{\mathrm{yx}}
\end{aligned}
$$


The solution for the left-inverse is

$$
g_{A B}^{\mathrm{xy}}=a_{\mathrm{xy}}^{l} \epsilon_{A C E}^{\mathrm{y}} \epsilon_{B D F}^{\mathrm{x}} g_{\mathrm{yx}}^{C D} g_{\mathrm{yx}}^{E F},
$$

where

$$
g_{A B}^{\mathrm{xy}} g_{\mathrm{yx}}^{B A}=\delta_{A}^{A} \Longrightarrow a_{\mathrm{xy}}^{l}=\frac{1}{2} \frac{\Delta_{\mathrm{yx}}}{\sqrt{\Delta_{\mathrm{y}} \Delta_{\mathrm{x}}}} .
$$

For the right-inverse it is

$$
g_{A B}^{\mathrm{yx}}=a_{\mathrm{yx}}^{r} \epsilon_{A C E}^{\mathrm{y}} \epsilon_{B D F}^{\mathrm{x}} g_{\mathrm{yx}}^{C D} g_{\mathrm{yx}}^{E F},
$$

where

$$
g_{\mathrm{xy}}^{A B} g_{B A}^{\mathrm{yx}}=\delta_{A}^{A} \Longrightarrow a_{\mathrm{xy}}^{r}=\frac{1}{2} \frac{\Delta_{\mathrm{xy}}}{\sqrt{\Delta_{\mathrm{x}} \Delta_{\mathrm{y}}}}
$$

Finally, we see that

$$
g_{A B}^{\mathrm{xy}}=g_{B A}^{\mathrm{yx}} .
$$

\section{Matter Space Covariance}

Because of the way we set up the worldline labeling - they are assigned, arbitrarily, on some timelike slice (cf. Fig. 1) — we can assert that there should be a relabeling invariance in the pull-back formalism. To that end, suppose we choose a new labeling scheme; e.g. we use three scalars $Y_{\mathrm{x}}^{A}$ to mark individual fluid worldlines. However, this process must be constrained in the sense that it only changes the label of a given worldline, and does not map to a different worldline. Clearly this process is a mapping $F_{\mathrm{x}}^{A}$ from the $X_{\mathrm{x}}^{A}$ to the $Y_{\mathrm{x}}^{A}$; i.e.

$$
Y_{\mathrm{x}}^{A}=F_{\mathrm{x}}^{A}\left\{X_{\mathrm{x}}^{B}\right\}
$$

Thus, the re-labeling of a worldline can be done, say, at $\left\{0, x_{\mathrm{x}}^{i}\left(\tau_{0}\right)\right\}$, where

$$
Y_{\mathrm{x}}^{A}\left[0, x_{\mathrm{x}}^{i}\left(\tau_{0}\right)\right]=F^{A}\left\{X_{\mathrm{x}}^{B}\left[0, x_{\mathrm{x}}^{i}\left(\tau_{0}\right)\right]\right\} .
$$

Finally, the constancy of the $Y_{\mathrm{x}}^{A}$ along the worldline is preserved by the mapping since

$$
\frac{\mathrm{d} Y_{\mathrm{x}}^{A}}{\mathrm{~d} \tau}=\frac{\partial F_{\mathrm{x}}^{A}}{\partial X_{\mathrm{x}}^{B}} \frac{\mathrm{d} X_{\mathrm{x}}^{B}}{\mathrm{~d} \tau}=0 .
$$

In principle, the $n_{A B C}^{\mathrm{x}}$ can have a functional dependence, say, of the $X_{\mathrm{x}}^{A}$ for each of the $N_{c}$ constituents as well as all the $g_{\mathrm{x}}^{A B}$ and $g_{\mathrm{xy}}^{A B}$. The mapping $F_{\mathrm{x}}^{A}$ for the worldline at $\left\{0, x_{\mathrm{x}}^{i}\left(\tau_{0}\right)\right\}$ must be such that

$$
n_{a b c}^{\mathrm{x}}\left\{Y_{\mathrm{x}}^{A}\left[0, x_{\mathrm{x}}^{i}\left(\tau_{0}\right)\right], \ldots\right\}=n_{a b c}^{\mathrm{x}}\left\{X_{\mathrm{x}}^{A}\left[0, x_{\mathrm{x}}^{i}\left(\tau_{0}\right)\right], \ldots\right\}
$$


where the new matter space metric components are

$$
\begin{aligned}
\bar{g}_{\mathrm{x}}^{A B} & \equiv \frac{\partial Y_{\mathrm{x}}^{A}}{\partial x^{a}} \frac{\partial Y_{\mathrm{x}}^{B}}{\partial x^{b}} g^{a b} \\
& =\frac{\partial F_{\mathrm{x}}^{A}}{\partial X_{\mathrm{x}}^{C}} \frac{\partial F_{\mathrm{x}}^{B}}{\partial X_{\mathrm{x}}^{D}} \frac{\partial X_{\mathrm{x}}^{C}}{\partial x^{a}} \frac{\partial X_{\mathrm{x}}^{D}}{\partial x^{b}} g^{a b} \\
& =\frac{\partial F_{\mathrm{x}}^{A}}{\partial X_{\mathrm{x}}^{C}} \frac{\partial F_{\mathrm{x}}^{B}}{\partial X_{\mathrm{x}}^{D}} g_{\mathrm{x}}^{C D}
\end{aligned}
$$

and

$$
\bar{g}_{\mathrm{xy}}^{A B} \equiv \frac{\partial Y_{\mathrm{x}}^{A}}{\partial x^{a}} \frac{\partial Y_{\mathrm{y}}^{B}}{\partial x^{b}} g^{a b}=\frac{\partial F_{\mathrm{x}}^{A}}{\partial X_{\mathrm{x}}^{C}} \frac{\partial F_{\mathrm{y}}^{B}}{\partial X_{\mathrm{y}}^{D}} g_{\mathrm{xy}}^{C D} .
$$

By rewriting Eq. (39), we find

$$
\begin{aligned}
0 & =\bar{n}_{A B C}^{\mathrm{x}} \frac{\partial Y_{\mathrm{x}}^{A}}{\partial x^{a}} \frac{\partial Y_{\mathrm{x}}^{B}}{\partial x^{b}} \frac{\partial Y_{\mathrm{x}}^{C}}{\partial x^{c}}-n_{A B C}^{\mathrm{x}} \frac{\partial X_{\mathrm{x}}^{A}}{\partial x^{a}} \frac{\partial X_{\mathrm{x}}^{B}}{\partial x^{b}} \frac{\partial Y_{\mathrm{x}}^{C}}{\partial x_{\mathrm{x}}^{c}} \\
& =\left(\frac{\partial F_{\mathrm{x}}^{D}}{\partial X_{\mathrm{x}}^{A}} \frac{\partial F_{\mathrm{x}}^{E}}{\partial X_{\mathrm{x}}^{B}} \frac{\partial F_{\mathrm{x}}^{F}}{\partial X_{\mathrm{x}}^{C}} \bar{n}_{D E F}^{\mathrm{x}}-n_{A B C}^{\mathrm{x}}\right) \frac{\partial X_{\mathrm{x}}^{A}}{\partial x^{a}} \frac{\partial X_{\mathrm{x}}^{B}}{\partial x_{\mathrm{x}}^{b}} \frac{\partial X_{\mathrm{x}}^{C}}{\partial x^{c}} \\
& \Longrightarrow n_{A B C}^{\mathrm{x}}=\frac{\partial F_{\mathrm{x}}^{D}}{\partial X_{\mathrm{x}}^{A}} \frac{\partial F_{\mathrm{x}}^{E}}{\partial X_{\mathrm{x}}^{B}} \frac{\partial F_{\mathrm{x}}^{F}}{\partial X_{\mathrm{x}}^{C}} \bar{n}_{D E F}^{\mathrm{x}},
\end{aligned}
$$

where

$$
\bar{n}_{A B C}^{\mathrm{x}}=\bar{n}_{A B C}^{\mathrm{x}}\left(F_{\mathrm{x}}^{A}, \frac{\partial F_{\mathrm{x}}^{A}}{\partial X_{\mathrm{x}}^{C}} \frac{\partial F_{\mathrm{x}}^{B}}{\partial X_{\mathrm{x}}^{D}} g_{\mathrm{x}}^{C D}, \ldots\right) .
$$

It has been asserted that Galilean invariance does not allow for $X_{\mathrm{x}}^{A}$ (via $\mathcal{N}_{\mathrm{x}}$ ) dependence in $n_{A B C}^{\mathrm{x}}$. But, we see that general mappings exist which preserve the covariance of the description, even those of a "translation" in matter space. This is important for what

follows later, since the resistivity enters precisely because we allow for the full set of $n_{A B C}^{\mathrm{x}}$ to depend, in principle, on all of the $X_{\mathrm{x}}^{A}$.

\section{THE ACTION PRINCIPLE, FIELD EQUATIONS, AND GAUGE INVARI-} ANCE

In this section we will set up an action principle to derive the resistive-fluid, Maxwell, and Einstein set of field equations. The pull-back formalism will be used to set up variations of the fluid fluxes $n_{\mathrm{x}}^{a}$ required to get the fluid equations with resistivity. The Maxwell equations are obtained by varying $A_{a}$, which appears in two pieces of the total action: one built from the antisymmetric, Faraday tensor $F_{a b}$, defined as

$$
F_{a b}=\nabla_{a} A_{b}-\nabla_{b} A_{a}
$$


and which satisfies a "Bianchi" identity

$$
0=\nabla_{a} F_{b c}+\nabla_{c} F_{a b}+\nabla_{b} F_{c a}
$$

and the other constructed from a coupling term based on the scalar $j_{\mathrm{x}}^{a} A_{a}$. Finally, the stressenergy tensor $T_{a b}$ is obtained in the usual way by varying the total action with respect to the metric $g_{a b}$. The Einstein field equations are obtained from the standard Hilbert action [21]. As these equations take the usual form, and our main focus is on the fluid dynamics, we will not discuss the gravitational degrees of freedom here. However, we do provide the full stress-tensor and so the Einstein equations follow immediately. Of course, it is important to note that the formalism does account for coupling of the fluid to the dynamical spacetime. The resistive fluid action $S_{M}$ (ignore boundary terms throughout) has as its Lagrangian an energy functional $\Lambda$ (also refereed to as the Master function). To motivate what it is we will need to review some basic thermodynamics. Much of this discussion is in Andersson and Comer [1], so we will try to limit the presentation here to the highlights.

Recall that thermodynamics allows one to describe a system with a large number of particles in terms of a small number of state variables. For a one-fluid system at nonzero temperature, each fluid element will have as its state variables the total number of particles $N$, the total entropy $S$, and the total volume $V$. With an equation of state, $E=E(N, S, V)$, the remaining thermodynamic variables of chemical potential $\mu$, pressure $p$, and the temperature $T$ are calculable as functions of the state variables. This is seen from the combined First and Second Laws of Thermodynamics:

$$
\mathrm{d} E=\mu \mathrm{d} N-p \mathrm{~d} V+T \mathrm{~d} S
$$

which implies

$$
\mu(N, S, V)=\frac{\partial E}{\partial N} \quad, \quad p(N, S, V)=\frac{\partial E}{\partial V} \quad, \quad T(N, S, V)=\frac{\partial E}{\partial S}
$$

We assume that the total energy and the state variables are extensive in the sense that if $(N, S, V)$ are doubled, say, then $E$ is also doubled. We also assume that the derived variables $(\mu, p, T)$ are intensive in that their values remain the same in the doubling process. These assumptions result in the Gibbs-Duhem (or Euler) relation [22]; namely,

$$
E+p V=\mu N+T S .
$$


Now we introduce the densities $n_{\mathrm{n}}=N / V, n_{\mathrm{s}}=S / V$, and $\Lambda=-E / V$, then Eqs. (46) and (48) lead to

$$
\begin{aligned}
\mathrm{d} \Lambda & =-\frac{\mu^{\mathrm{n}}}{2 n_{\mathrm{n}}} \mathrm{d} n_{\mathrm{n}}^{2}-\frac{\mu^{\mathrm{s}}}{2 n_{\mathrm{s}}} \mathrm{d} n_{\mathrm{s}}^{2} \\
\mu^{\mathrm{n}}\left(n_{\mathrm{n}}^{2}, n_{\mathrm{s}}^{2}\right) & =-2 n_{\mathrm{n}} \frac{\partial \Lambda}{\partial n_{\mathrm{n}}^{2}} \equiv \mathcal{B}^{\mathrm{n}} n_{\mathrm{n}}, \\
\mu^{\mathrm{s}}\left(n_{\mathrm{n}}^{2}, n_{\mathrm{s}}^{2}\right) & =-2 n_{\mathrm{s}} \frac{\partial \Lambda}{\partial n_{\mathrm{s}}^{2}} \equiv \mathcal{B}^{\mathrm{s}} n_{\mathrm{s}} \\
\Psi\left(n_{\mathrm{n}}^{2}, n_{\mathrm{s}}^{2}\right) & =\Lambda+\mathcal{B}^{\mathrm{n}} n_{\mathrm{n}}^{2}+\mathcal{B}^{\mathrm{s}} n_{\mathrm{s}}^{2}
\end{aligned}
$$

where we have put in place the notational conventions established earlier. It is essential to note that the fundamental thermodynamic state variables are now $\left(n_{\mathrm{n}}^{2}, n_{\mathrm{s}}^{2}\right)$, the equation of state is the, a priori, known function $\Lambda\left(n_{\mathrm{n}}^{2}, n_{\mathrm{s}}^{2}\right)$, and the remaining thermodynamic quantities of particle number chemical potential $\mu_{\mathrm{n}}$, temperature $\mu_{\mathrm{s}}$, and the pressure $\Psi$ are calculable from $\Lambda$.

Finally, we introduce the fluid four-velocity $u^{a}$ (where $u^{a} u_{a}=-1$ ) for our single fluid system. The two fluid fluxes become $n_{\mathrm{n}}^{a}=n_{\mathrm{n}} u^{a}$ and $n_{\mathrm{s}}^{a}=n_{\mathrm{s}} u^{a}$ and we are to understand that the densities are obtained from them as $n_{\mathrm{x}}^{2}=-n_{a}^{\mathrm{x}} n_{\mathrm{x}}^{a}$, where $\mathrm{x}=\{\mathrm{n}, \mathrm{s}\}$. We can now see that the combined First and Second Law of Thermodynamics takes the form

$$
\mathrm{d} \Lambda=\mu_{a}^{\mathrm{n}} \mathrm{d} n_{\mathrm{n}}^{a}+\mu_{a}^{\mathrm{s}} \mathrm{d} n_{\mathrm{s}}^{a}
$$

and we have the emergence of the chemical potential covectors thermodynamically conjugate to the fluxes:

$$
\mu_{a}^{\mathrm{n}}=\mathcal{B}^{\mathrm{n}} n_{a}^{\mathrm{n}} \quad, \quad \mu_{a}^{\mathrm{s}}=\mathcal{B}^{\mathrm{s}} n_{a}^{\mathrm{s}}
$$

In the same spirit, the pressure $\Psi$ is

$$
\Psi=\Lambda-\mu_{a}^{\mathrm{n}} n_{\mathrm{n}}^{a}-\mu_{a}^{\mathrm{s}} n_{\mathrm{s}}^{a}
$$

Therefore, we have shown that given a form for $\Lambda\left(n_{\mathrm{n}}^{2}, n_{\mathrm{s}}^{2}\right)$, and acquiring the fluxes $n_{\mathrm{x}}^{a}$, say, from solutions to the field equations, we can determine completely the remaining set of thermodynamic variables: chemical potential, temperature, and pressure.

In the following section, we will consider a system with an arbitrary number of independent fluid flows. Guided by analogies with superfluids, and ordinary heat conducting fluids, we know that the energy functional must be expanded to allow for entrainment (see 
[1] for details). Mathematically, this means we include in $\Lambda$ a dependence on all the scalars formed from inner products of different fluid fluxes; i.e. terms of the form $n_{\mathrm{xy}}^{2}=-n_{a}^{\mathrm{x}} n_{\mathrm{y}}^{a}$, where $\mathrm{x} \neq \mathrm{y}$.

\section{A. The Matter, Electromagnetic, and Coupling Actions}

The fluid action $S_{M}$ (ignore boundary terms throughout) has as its Lagrangian the functional $\Lambda$, and we know from the previous section that it depends on the $n_{\mathrm{x}}^{2}=-n_{a}^{\mathrm{x}} n_{\mathrm{x}}^{a}$ and the $n_{\mathrm{xy}}^{2}=-g_{a b} n_{\mathrm{x}}^{a} n_{\mathrm{y}}^{b}$. An arbitrary variation of $S_{M}$ with respect to the fluxes $n_{\mathrm{x}}^{a}$ and the metric gives

$$
\begin{aligned}
\delta S_{M} & =\delta\left(\int_{\mathcal{M}} \mathrm{d}^{4} x \sqrt{-g} \Lambda\right) \\
& =\int_{\mathcal{M}} \mathrm{d}^{4} x \sqrt{-g}\left[\sum_{\mathrm{x}} \mu_{a}^{\mathrm{x}} \delta n_{\mathrm{x}}^{a}+\frac{1}{2}\left(\Lambda g^{a b}+\sum_{\mathrm{x}} n_{\mathrm{x}}^{a} \mu_{\mathrm{x}}^{b}\right) \delta g_{a b}\right],
\end{aligned}
$$

where $g$ is the determinant of the metric and $\mu_{a}^{\mathrm{x}}$ is the canonically conjugate momentum to $n_{\mathrm{x}}^{a}$; that is, letting

$$
\mathcal{B}^{\mathrm{x}}=-2 \frac{\partial \Lambda}{\partial n_{\mathrm{x}}^{2}} \quad, \quad \mathcal{A}^{\mathrm{xy}}=-\frac{\partial \Lambda}{\partial n_{\mathrm{xy}}^{2}}
$$

then

$$
\mu_{a}^{\mathrm{x}}=g_{a b}\left(\mathcal{B}^{\mathrm{x}} n_{\mathrm{x}}^{b}+\sum_{\mathrm{y} \neq \mathrm{x}} \mathcal{A}^{\mathrm{xy}} n_{\mathrm{y}}^{b}\right) .
$$

As mentioned earlier, the momentum is an essential piece of the formalism. For example, the antisymmetric vorticity two-form $\omega_{a b}^{\mathrm{x}}$ is obtained as the exterior derivative of $\mu_{a}^{\mathrm{x}}$; that is,

$$
\omega_{a b}^{\mathrm{x}}=2 \nabla_{[a} \mu_{b]}^{\mathrm{x}} .
$$

Its role as vorticity is well established; e.g. when $\mu_{a}^{\mathrm{x}}$ is the gradient of a scalar then $\omega_{a b}^{\mathrm{x}}$ is zero (as is the case for superfluids). Notice also how the inclusion of $n_{\mathrm{xy}}^{2}$ has led to so-called "entrainment", a tilting of the fluid momenta in the sense that $\mu_{a}^{\mathrm{x}}$ is no longer simply proportional to its own flux $n_{\mathrm{x}}^{a}$. Entrainment [23-26] between neutrons and protons is generally thought to be important in superfluid neutron stars and entrainment between matter and entropy can be shown to be important for causal heat conductivity [27].

The Maxwell Action is

$$
S_{M a x}=\frac{1}{16 \pi} \int_{\mathcal{M}} \mathrm{d}^{4} x \sqrt{-g} F_{a b} F^{a b}
$$


and its variation with respect to $A_{a}$ and the metric $g_{a b}$ is

$$
\delta S_{M a x}=\frac{1}{4 \pi} \int_{\mathcal{M}} \mathrm{d}^{4} x \sqrt{-g}\left(\nabla_{a} F^{a b}\right) \delta A_{b}-\frac{1}{32 \pi} \int_{\mathcal{M}} \mathrm{d}^{4} x \sqrt{-g}\left(F_{c d} F^{c d} g^{a b}-4 F^{a c} F^{b}\right) \delta g_{a b} .
$$

The minimal coupling of the Maxwell field to the charge current densities is obtained from the Coulomb action

$$
S_{C}=\int_{\mathcal{M}} \mathrm{d}^{4} x \sqrt{-g} \sum_{\mathrm{x}} j_{\mathrm{x}}^{a} A_{a}
$$

whose variation with respect to $n_{\mathrm{x}}^{a}, A_{a}$, and $g_{a b}$ gives

$$
\delta S_{C}=\int_{\mathcal{M}} \mathrm{d}^{4} x \sqrt{-g} \sum_{\mathrm{x}}\left(j_{\mathrm{x}}^{a} \delta A_{a}+e_{\mathrm{x}} A_{a} \delta n_{\mathrm{x}}^{a}+\frac{1}{2} j_{\mathrm{x}}^{a} A_{a} g^{b c} \delta g_{b c}\right)
$$

The variation of the total action $S$ for the system is thus

$$
\begin{aligned}
\delta S & =\delta S_{M}+\delta S_{\text {Max }}+\delta S_{C} \\
& =\int_{\mathcal{M}} \mathrm{d}^{4} x \sqrt{-g}\left\{\sum_{\mathrm{x}}\left(\mu_{a}^{\mathrm{x}}+e_{\mathrm{x}} A_{a}\right) \delta n_{\mathrm{x}}^{a}+\frac{1}{4 \pi}\left(\nabla_{b} F^{b a}+4 \pi \sum_{\mathrm{x}} j_{\mathrm{x}}^{a}\right) \delta A_{a}\right. \\
& \left.+\frac{1}{2}\left[\Lambda g^{a b}+\sum_{\mathrm{x}}\left(n_{\mathrm{x}}^{a} \mu_{\mathrm{x}}^{b}+j_{\mathrm{x}}^{c} A_{c} g^{a b}\right)-\frac{1}{16 \pi}\left(F_{c d} F^{c d} g^{a b}-4 F^{a c} F^{b}{ }_{c}\right)\right] \delta g_{a b}\right\} .
\end{aligned}
$$

Note that the minimal coupling has given a modification of the conjugate momentum familiar from, say, quantum mechanics; namely,

$$
\tilde{\mu}_{a}^{\mathrm{x}}=\mu_{a}^{\mathrm{x}}+e_{\mathrm{x}} A_{a}
$$

Of course, the field equations obtained from the variation above cannot be the final form, since the term proportional to $\delta n_{\mathrm{x}}^{a}$ implies that the momentum $\tilde{\mu}_{a}^{\mathrm{x}}$ must vanish. Clearly this is not viable because it is essentially the condition that there be no energy or matter present. This occurs because the components of $\delta n_{\mathrm{x}}^{a}$ cannot all be varied independently. We need a constrained variation. The pull-back formalism provides a set of alternative variables which does precisely that - the $X_{\mathrm{x}}^{A}$ can be varied independently. Also, we have to incorporate the fact that the fluid momentum has changed from $\mu_{a}^{\mathrm{x}}$ to $\tilde{\mu}_{a}^{\mathrm{x}}$. This is straightforward since all that is required is to take Eq. (5) and replace $\mu_{a}^{\mathrm{x}}$ with $\tilde{\mu}_{a}^{\mathrm{x}}, \mu_{a b c}^{\mathrm{x}}$ with $\tilde{\mu}_{a b c}^{\mathrm{x}}$, and use that, as well as $\tilde{\mu}^{\mathrm{x}}=-u_{\mathrm{x}}^{a} \tilde{\mu}_{a}^{\mathrm{x}}$, as the basis for what follows below.

\section{B. Lagrangian Displacements}

Even though we have as our unconstrained dynamical variables the scalars $X_{\mathrm{x}}^{A}$, ultimately we want the action principle to produce field equations for the fluxes. After all, there 
is decades of literature and computational techniques for fluids based on solving for the components $n_{\mathrm{x}}^{a}$ and not the $X_{\mathrm{x}}^{A}$. Fortunately, we can use Lagrangian displacements to bridge variations of matter space variables to those of spacetime. Denoted $\xi_{\mathrm{x}}^{a}$, the Lagrangian displacement for a fluid needs to be such that it tracks the virtual displacements of fluid element worldlines in spacetime. And even though it is a spacetime vector, with four components, it has been shown (see [1]) that there is gauge freedom that reduces the number of degrees of freedom back to three.

Using the standard definition of a Lagrangian variation in the relativistic context [1], we write

$$
\Delta_{\mathrm{x}} X_{\mathrm{x}}^{A}=\delta X_{\mathrm{x}}^{A}+\mathcal{L}_{\xi_{\mathrm{x}}} X_{\mathrm{x}}^{A}=0
$$

where $\delta X_{\mathrm{x}}^{A}$ is the Eulerian variation and $\mathcal{L}_{\xi_{\mathrm{x}}}$ is the Lie derivative. This means that convective variations are such that

$$
\delta X_{\mathrm{x}}^{A}=-\mathcal{L}_{\xi_{\mathrm{x}}} X_{\mathrm{x}}^{A}=-\xi_{\mathrm{x}}^{a} \frac{\partial X_{\mathrm{x}}^{A}}{\partial x^{a}}
$$

The displacements of the matter space fluid elements will lead to variations of $n_{A B C}^{\mathrm{x}}$, which, in turn, will induce variations of $n_{a b c}^{\mathrm{x}}$. The existence of more than one fluid means, also, that we need to consider

$$
\Delta_{\mathrm{x}} X_{\mathrm{y}}^{A}=\delta X_{\mathrm{y}}^{A}+\mathcal{L}_{\xi_{\mathrm{x}}} X_{\mathrm{y}}^{A}=\mathcal{L}_{\xi_{\mathrm{x}}} X_{\mathrm{y}}^{A}-\mathcal{L}_{\xi_{\mathrm{y}}} X_{\mathrm{y}}^{A}=\left(\xi_{\mathrm{x}}^{a}-\xi_{\mathrm{y}}^{a}\right) \frac{\partial X_{\mathrm{y}}^{A}}{\partial x^{a}}
$$

The Lagrangian variation of $n_{a b c}^{\mathrm{x}}$ in general is

$$
\Delta_{\mathrm{x}} n_{a b c}^{\mathrm{x}}={ }^{\mathrm{x}} \mathcal{J}_{a b c}^{A B C} \Delta_{\mathrm{x}} n_{A B C}^{\mathrm{x}}
$$

and thus

$$
\delta n_{a b c}^{\mathrm{x}}=-\mathcal{L}_{\xi_{\mathrm{x}}} n_{a b c}^{\mathrm{x}}+{ }^{\mathrm{x}} \mathcal{J}_{a b c}^{A B C} \Delta_{\mathrm{x}} n_{A B C}^{\mathrm{x}}
$$

where the Lie derivative of the $n_{a b c}^{\mathrm{x}}$ along the $\xi_{\mathrm{x}}^{a}$ is

$$
\mathcal{L}_{\xi_{\mathrm{x}}} n_{a b c}^{\mathrm{x}}=\xi_{\mathrm{x}}^{d} \frac{\partial n_{a b c}^{\mathrm{x}}}{\partial x^{d}}+n_{d b c}^{\mathrm{x}} \frac{\partial \xi_{\mathrm{x}}^{d}}{\partial x^{a}}+n_{a d c}^{\mathrm{x}} \frac{\partial \xi_{\mathrm{x}}^{d}}{\partial x^{b}}+n_{a b d}^{\mathrm{x}} \frac{\partial \xi_{\mathrm{x}}^{d}}{\partial x^{c}}
$$

Andersson and Comer [16] have demonstrated that allowing $n_{A B C}^{\mathrm{x}}$ to be a function of all the $X_{\mathrm{x}}^{A}$ (including $X_{\mathrm{y}}^{A}$ for $\mathrm{y} \neq \mathrm{x}$ ), all the $g_{\mathrm{x}}^{A B}$, and all the $g_{\mathrm{xy}}^{A B}$ leads to a system of fluid equations with dissipation of several types, among which is the resistive type of interactions to be explored here and others coming from, say, shear and bulk viscosities. The resistive 
forms of dissipation are due to the presence of $X_{\mathrm{y}}^{A}($ for $\mathrm{y} \neq \mathrm{x})$ in the $n_{A B C}^{\mathrm{x}}$, and so we consider here

$$
\begin{aligned}
\Delta_{\mathrm{x}} n_{A B C}^{\mathrm{x}} & =\sum_{\mathrm{y} \neq \mathrm{x}} \frac{\partial n_{A B C}^{\mathrm{x}}}{\partial X_{\mathrm{y}}^{D}} \Delta_{\mathrm{x}} X_{\mathrm{y}}^{D} \\
& =\sum_{\mathrm{y} \neq \mathrm{x}} \frac{\partial n_{A B C}^{\mathrm{x}}}{\partial X_{\mathrm{y}}^{D}} \frac{\partial X_{\mathrm{y}}^{D}}{\partial x^{a}}\left(\xi_{\mathrm{x}}^{a}-\xi_{\mathrm{y}}^{a}\right) .
\end{aligned}
$$

Using the facts that

$$
\begin{aligned}
& \Delta_{\mathrm{x}} g^{a b}=\delta g^{a b}-2 \nabla^{(a} \xi_{\mathrm{x}}^{b)}, \\
& \delta \epsilon^{a b c d}=-\frac{1}{2} \epsilon^{a b c d} g^{e f} \delta g_{e f},
\end{aligned}
$$

and

$$
\epsilon^{b c d a} \mathcal{L}_{\xi_{\mathrm{x}}} n_{b c d}^{\mathrm{x}}=3 !\left(\xi_{\mathrm{x}}^{b} \nabla_{b} n_{\mathrm{x}}^{a}-n_{\mathrm{x}}^{b} \nabla_{b} \xi_{\mathrm{x}}^{a}+n_{\mathrm{x}}^{a} \nabla_{b} \xi_{\mathrm{x}}^{b}\right)
$$

we find

$$
\begin{aligned}
\delta n_{\mathrm{x}}^{a} & =\delta\left(\frac{1}{3 !} \epsilon^{b c d a} n_{b c d}^{\mathrm{x}}\right) \\
& =n_{\mathrm{x}}^{b} \nabla_{b} \xi_{\mathrm{x}}^{a}-\xi_{\mathrm{x}}^{b} \nabla_{b} n_{\mathrm{x}}^{a}-n_{\mathrm{x}}^{a}\left(\nabla_{b} \xi_{\mathrm{x}}^{b}+\frac{1}{2} g^{b c} \delta g_{b c}\right)+\frac{1}{\tilde{\mu}^{\mathrm{x}} n_{\mathrm{x}}} n_{\mathrm{x}}^{a} \sum_{\mathrm{y} \neq \mathrm{x}} R_{b}^{\mathrm{xy}}\left(\xi_{\mathrm{x}}^{b}-\xi_{\mathrm{y}}^{b}\right),
\end{aligned}
$$

where

$$
R_{a}^{\mathrm{xy}} \equiv \frac{1}{3 !} \frac{\partial X_{\mathrm{y}}^{D}}{\partial x^{a}} \tilde{\mu}_{\mathrm{x}}^{A B C} \frac{\partial n_{A B C}^{\mathrm{x}}}{\partial X_{\mathrm{y}}^{D}}
$$

and it satisfies the identity

$$
u_{\mathrm{y}}^{a} R_{a}^{\mathrm{xy}} \equiv 0
$$

The total "resistivity" current $R_{a}^{\mathrm{x}}$ is

$$
R_{a}^{\mathrm{x}}=\sum_{\mathrm{y} \neq \mathrm{x}}\left(R_{a}^{\mathrm{yx}}-R_{a}^{\mathrm{xy}}\right)
$$

which has the identity

$$
\sum_{\mathrm{x}} R_{a}^{\mathrm{x}} \equiv 0
$$

\section{The Field Equations}

We now return to the flux variations of the combined fluid, Coulomb, and Maxwell actions given in Eq. (64). The fact that we are summing over all constituents leads to

$$
\sum_{\mathrm{x}} \sum_{\mathrm{y} \neq \mathrm{x}} R_{a}^{\mathrm{xy}}\left(\xi_{\mathrm{x}}^{a}-\xi_{\mathrm{y}}^{a}\right)=\sum_{\mathrm{x}} \sum_{\mathrm{y} \neq \mathrm{x}}\left(R_{a}^{\mathrm{xy}}-R_{a}^{\mathrm{yx}}\right) \xi_{\mathrm{x}}^{a}=-\sum_{\mathrm{x}} R_{a}^{\mathrm{x}} \xi_{\mathrm{x}}^{a},
$$


so that the variation of the action (excluding the Hilbert action for reasons stated earlier) for the system is

$$
\begin{aligned}
\delta S= & \int_{\mathcal{M}} \mathrm{d}^{4} x \sqrt{-g}\left\{-\sum_{\mathrm{x}}\left(f_{a}^{\mathrm{x}}+\Gamma_{\mathrm{x}} \tilde{\mu}_{a}^{\mathrm{x}}-R_{a}^{\mathrm{x}}\right) \xi_{\mathrm{x}}^{a}-\frac{1}{4 \pi}\left(\nabla_{b} F^{a b}-4 \pi \sum_{\mathrm{x}} j_{\mathrm{x}}^{a}\right) \delta A_{a}\right. \\
& \left.+\frac{1}{2}\left[\Psi g^{a b}+\sum_{\mathrm{x}} n_{\mathrm{x}}^{a} \mu_{\mathrm{x}}^{b}-\frac{1}{16 \pi}\left(F_{c d} F^{c d} g^{a b}-4 F^{a c} F^{b}{ }_{c}\right)\right] \delta g_{a b}\right\} .
\end{aligned}
$$

where

$$
\begin{gathered}
f_{a}^{\mathrm{x}}=n_{\mathrm{x}}^{b} \tilde{\omega}_{b a}^{\mathrm{x}} \equiv 2 n_{\mathrm{x}}^{b} \nabla_{[a} \tilde{\mu}_{b]}^{\mathrm{x}}, \\
\Gamma_{\mathrm{x}}=\nabla_{a} n_{\mathrm{x}}^{a},
\end{gathered}
$$

and

$$
\Psi=\Lambda-\sum_{\mathrm{x}} \mu_{c}^{\mathrm{x}} n_{\mathrm{x}}^{c}
$$

The momentum equation for each fluid is

$$
f_{a}^{\mathrm{x}}+\Gamma_{\mathrm{x}} \tilde{\mu}_{a}^{\mathrm{x}}=R_{a}^{\mathrm{x}}
$$

the Maxwell equation (including also Eq. (45)) is

$$
\nabla_{b} F^{a b}=\nabla_{b}\left(\nabla^{a} A^{b}-\nabla^{b} A^{a}\right)=4 \pi \sum_{\mathrm{x}} j_{\mathrm{x}}^{a}
$$

and the stress-energy tensor is

$$
T^{a b}=\Psi g^{a b}+\sum_{\mathrm{x}} n_{\mathrm{x}}^{a} \mu_{\mathrm{x}}^{b}-\frac{1}{16 \pi}\left(F_{c d} F^{c d} g^{a b}-4 F^{a c} F_{c}^{b}\right) .
$$

\section{Impact of Change of Gauge for $A_{a}$}

An electromagnetic gauge transformation will impact the fluid equations of motion because of the change to the momentum; i.e. letting $\bar{A}_{a}=A_{a}+\nabla_{a} \phi$ we find

$$
\tilde{\mu}_{a}^{\mathrm{x}}=\mu_{a}^{\mathrm{x}}+e_{\mathrm{x}} A_{a} \quad \longrightarrow \quad \bar{\mu}_{a}^{\mathrm{x}}=\mu_{a}^{\mathrm{x}}+e_{\mathrm{x}} \bar{A}_{a}=\tilde{\mu}_{a}^{\mathrm{x}}+e_{\mathrm{x}} \nabla_{a} \phi
$$

It is important here to consider in more detail the ramifications of such a change of gauge, since an application of the present work will be to numerical evolutions [18]. In the numerical setting, we expect to be solving for the vector potential $A_{a}$ as we evolve the system. This

will require a choice of gauge for the vector potential, which will affect the explicit values of terms (such as the resistivity) in the equations of motion. 
In Eq. (86) (i.e. the fluid equation of motion), we see the term involving $\Gamma_{\mathrm{x}}$ is changed but not $f_{a}^{\mathrm{x}}$. What also changes is $R_{a}^{\mathrm{x}}$, since the quantity $\tilde{\mu}_{\mathrm{x}}^{A B C}$ in $R_{a}^{\mathrm{xy}}[c f$. Eq. (77)] depends on $A_{a}$. Letting $\bar{R}_{a}^{\mathrm{x}}$ denote the resistivity in the new gauge, we find

$$
\begin{aligned}
\bar{R}_{a}^{\mathrm{x}}= & \sum_{\mathrm{y} \neq \mathrm{x}}\left(\bar{R}_{a}^{\mathrm{yx}}-\bar{R}_{a}^{\mathrm{xy}}\right) \\
= & \sum_{\mathrm{y} \neq \mathrm{x}} \frac{1}{3 !} \epsilon^{e b c d}\left[\left(\tilde{\mu}_{e}^{\mathrm{y}}+e_{\mathrm{y}} \nabla_{e} \phi\right)^{\mathrm{y}} \mathcal{J}_{b c d}^{A B C} \frac{\partial n_{A B C}^{\mathrm{y}}}{\partial X_{\mathrm{x}}^{D}} \frac{\partial X_{\mathrm{x}}^{D}}{\partial x^{a}}\right. \\
& \left.-\left(\tilde{\mu}_{e}^{\mathrm{x}}+e_{\mathrm{x}} \nabla_{e} \phi\right)^{\mathrm{x}} \mathcal{J}_{b c d}^{A B C} \frac{\partial n_{A B C}^{\mathrm{x}}}{\partial X_{\mathrm{y}}^{D}} \frac{\partial X_{\mathrm{y}}^{D}}{\partial x^{a}}\right] \\
= & R_{a}^{\mathrm{x}}+G_{a}^{\mathrm{x}}
\end{aligned}
$$

where

$$
G_{a}^{\mathrm{x}}=\sum_{\mathrm{y} \neq \mathrm{x}}\left(G_{a}^{\mathrm{yx}}-G_{a}^{\mathrm{xy}}\right) \quad, \quad G_{a}^{\mathrm{xy}}=\frac{1}{3 !} \epsilon^{e b c d} e_{\mathrm{x}}\left({ }^{\mathrm{x}} \mathcal{J}_{b c d}^{A B C} \frac{\partial n_{A B C}^{\mathrm{x}}}{\partial X_{\mathrm{y}}^{D}} \frac{\partial X_{\mathrm{y}}^{D}}{\partial x^{a}}\right) \nabla_{e} \phi
$$

Note that

$$
\sum_{\mathrm{x}} R_{a}^{\mathrm{x}}=\sum_{\mathrm{x}} G_{a}^{\mathrm{x}}=0 \Longrightarrow \sum_{\mathrm{x}} \bar{R}_{a}^{\mathrm{x}}=\sum_{\mathrm{x}} R_{a}^{\mathrm{x}}+\sum_{\mathrm{x}} G_{a}^{\mathrm{x}}=0 .
$$

In the new gauge the fluid equation of motion becomes

$$
\begin{aligned}
0 & =\bar{f}_{a}^{\mathrm{x}}+\Gamma_{\mathrm{x}} \bar{\mu}_{a}^{\mathrm{x}}+\bar{R}_{a}^{\mathrm{x}} \\
& =f_{a}^{\mathrm{x}}+\Gamma_{\mathrm{x}}\left(\tilde{\mu}_{a}^{\mathrm{x}}+e_{\mathrm{x}} \nabla_{a} \phi\right)-\left(R_{a}^{\mathrm{x}}+G_{a}^{\mathrm{x}}\right) .
\end{aligned}
$$

Projecting along $n_{\mathrm{x}}^{a}$ we find

$$
0=\Gamma_{\mathrm{x}} n_{\mathrm{x}}^{a}\left(\tilde{\mu}_{a}^{\mathrm{x}}+e_{\mathrm{x}} \nabla_{a} \phi\right)-n_{\mathrm{x}}^{a}\left(R_{a}^{\mathrm{x}}+G_{a}^{\mathrm{x}}\right)
$$

We have seen above that the observables, including the stress-energy tensor, Faraday tensor, and all hydrodynamic variables are independent of the choice of gauge for $A_{a}$, as expected. However, the fluid field equations are modified, which is also expected. Nevertheless, we can determine the modifications and thereby evolve the system regardless of the choice of gauge.

\section{E. Gauge Invariance and Charge Conservation}

To see other consequences of gauge invariance, we will consider a variation of the total action, where the vector potential variation takes the form

$$
\delta A_{a}=\nabla_{a} \delta \phi
$$


We assume that $\xi_{\mathrm{x}}^{a}=0$ and $\delta g_{a b}=0$ under the change of gauge; thus, even though the term $R_{a}^{\mathrm{xy}}$ acquires the gauge term $G_{a}^{\mathrm{xy}}\left[c f\right.$. Eq. (91)] it does not affect $\delta n_{\mathrm{x}}^{a}$. The total action thus reduces to

$$
\begin{aligned}
\delta S & =-\frac{1}{4 \pi} \int_{\mathcal{M}} \mathrm{d}^{4} x \sqrt{-g}\left(\nabla_{b} F^{a b}-4 \pi \sum_{\mathrm{x}} j_{\mathrm{x}}^{a}\right) \nabla_{a} \delta \phi \\
& =-\frac{1}{4 \pi} \int_{\mathcal{M}} \mathrm{d}^{4} x \sqrt{-g} \nabla_{a}\left(\nabla_{b} F^{a b}-4 \pi \sum_{\mathrm{x}} j_{\mathrm{x}}^{a}\right) \delta \phi,
\end{aligned}
$$

which implies

$$
\nabla_{a}\left(\nabla_{b} F^{a b}\right)=4 \pi \sum_{\mathrm{x}} e^{\mathrm{x}} \nabla_{a} n_{\mathrm{x}}^{a}=4 \pi \sum_{\mathrm{x}} e^{\mathrm{x}} \Gamma_{\mathrm{x}} .
$$

However, the commutation of covariant derivatives acting on a two-index object is

$$
\nabla_{a} \nabla_{b} F_{d}^{c}-\nabla_{b} \nabla_{a} F_{d}^{c}=R_{e a b}^{c} F_{d}^{e}-R_{d a b}^{e} F_{e}^{c}
$$

hence,

$$
\frac{1}{4 \pi} \nabla_{a}\left(\nabla_{b} F^{a b}\right)=\frac{1}{4 \pi} R_{a b} F^{a b} \equiv 0
$$

since the Ricci tensor is symmetric and the Faraday tensor is antisymmetric. Thus, we recover the expected conservation of charge:

$$
\sum_{\mathrm{x}} e^{\mathrm{x}} \Gamma_{\mathrm{x}}=\sum_{\mathrm{x}} \nabla_{a} j_{\mathrm{x}}^{a}=0
$$

Using the field equations, and Eqs. (80) and (100), we can show that $\nabla_{a} T^{a b}$ vanishes identically (as it should from diffeomorphism invariance):

$$
\begin{aligned}
\nabla_{b} T_{a}^{b} & =\nabla_{b}\left[\Psi \delta_{a}^{b}+\sum_{\mathrm{x}} n_{\mathrm{x}}^{b} \mu_{a}^{\mathrm{x}}-\frac{1}{16 \pi}\left(F_{c d} F^{c d} \delta_{a}^{b}-4 F^{b c} F_{a c}\right)\right] \\
& =\sum_{\mathrm{x}} R_{a}^{\mathrm{x}}+\left(\sum_{\mathrm{x}} e_{\mathrm{x}} \Gamma_{\mathrm{x}}\right) A_{a} \equiv 0 .
\end{aligned}
$$

\section{A PHENOMENOLOGICAL APPROACH TO THE RESISTIVITY}

Having completed the formal considerations, we can turn our attention to applications. As we do so, it is very important to appreciate that the $n_{A B C}^{\mathrm{x}}$ and how they enter $\Lambda$ is assumed to be "known" a priori. It is not until a specific application is intended that one would necessarily require an explicit relation. An analogy is the Lagrangian for an interacting 
complex scalar field. A potential $V\left(\phi^{\dagger} \phi\right)$ is introduced, but not generally specified until the Euler-Lagrange equations are derived and a specific application is pursued.

At this point, the action principle has given us the tensorial structure of the equations and how many different dissipative processes exist in general. Ideally, what we would do next is use microphysics to specify the $n_{A B C}^{\mathrm{x}}$ and $\Lambda$. Admittedly that task is daunting and would require more specifics about the actual systems to be described. Instead, we will develop here a phenomenological form of the resistivity $R_{a}^{\mathrm{x}}$, which is consistent with the field equations above, the various identities, and the Second Law of Thermodynamics.

To begin, it is convenient to introduce a fiducial frame-of-reference ${ }^{1}$ whose worldline is determined by the unit four-velocity $u^{a}$. Locally, we can decompose our fields into pieces parallel to $u^{a}$ and perpendicular to $u^{a}$ using the projection operator

$$
\perp_{b}^{a} \equiv \delta_{b}^{a}+u^{a} u_{b} \quad, \quad u_{a} u^{a}=-1
$$

For instance, the particle flux unit vectors are now decomposed as

$$
v_{\mathrm{x}}^{a} \equiv \gamma_{\mathrm{x}} \perp_{b}^{a} u_{\mathrm{x}}^{b} \quad \Longrightarrow \quad u_{\mathrm{x}}^{a}=\gamma_{\mathrm{x}}\left(u^{a}+v_{\mathrm{x}}^{a}\right) \quad, \quad \gamma_{\mathrm{x}}^{2}=\left(1-v_{\mathrm{x}}^{a} v_{a}^{\mathrm{x}}\right)^{-1}
$$

where $v_{\mathrm{x}}^{a}$ is the (coordinate-based time) three-velocity.

Recall that the resistivity is given by [cf. Eqs. (78) and (79)]

$$
R_{a}^{\mathrm{x}}=\sum_{\mathrm{y} \neq \mathrm{x}}\left(R_{a}^{\mathrm{yx}}-R_{a}^{\mathrm{xy}}\right) \quad, \quad u_{\mathrm{y}}^{a} R_{a}^{\mathrm{xy}}=0
$$

and $R_{a}^{\mathrm{xy}}$ is defined in Eq. (77). Its decomposition is

$$
\hat{R}_{a}^{\mathrm{xy}} \equiv \perp_{a}^{b} R_{b}^{\mathrm{xy}} \quad \Longrightarrow \quad R_{a}^{\mathrm{xy}}=\left(-u^{b} R_{b}^{\mathrm{xy}}\right) u_{a}+\hat{R}_{a}^{\mathrm{xy}}
$$

The constraint on $R_{a}^{\mathrm{xy}}[c f$. Eq. (78)] becomes

$$
\begin{aligned}
0= & u_{\mathrm{y}}^{a} R_{a}^{\mathrm{xy}}=\gamma_{\mathrm{y}}\left(u^{a}+v_{\mathrm{y}}^{a}\right) R_{a}^{\mathrm{xy}} \\
& \Longrightarrow-u^{a} R_{a}^{\mathrm{xy}}=v_{\mathrm{y}}^{a} \hat{R}_{a}^{\mathrm{xy}}
\end{aligned}
$$

thus, $R_{a}^{\mathrm{xy}}$ — for given $\mathrm{x}$ and $\mathrm{y}$ - has only 3 free components $\hat{R}_{a}^{\mathrm{xy}}$, and takes the form

$$
R_{a}^{\mathrm{xy}}=\left(\delta_{a}^{b}+v_{\mathrm{y}}^{b} u_{a}\right) \hat{R}_{b}^{\mathrm{xy}}
$$

\footnotetext{
${ }^{1}$ In an accompanying paper [17], we will consider a family of worldlines of this type and form a fibration of spacetime, and in [18] we will make use of a field $N^{a}$ which is surface-forming and hence can provide a foliation for a $3+1$ decomposition of spacetime.
} 
Putting all these pieces together, the total resistivity takes the form

$$
R_{a}^{\mathrm{x}}=\sum_{\mathrm{y} \neq \mathrm{x}}\left[\left(v_{\mathrm{x}}^{b} \hat{R}_{b}^{\mathrm{yx}}-v_{\mathrm{y}}^{b} \hat{R}_{b}^{\mathrm{xy}}\right) u_{a}+\hat{R}_{a}^{\mathrm{yx}}-\hat{R}_{a}^{\mathrm{xy}}\right]
$$

It is easy to see that the "time" and "space" pieces separately satisfy Eq. (80).

Using the fluid equations of motion we can relate the resistivity to the particle number creation rate $\Gamma_{\mathrm{x}}$. Note that Eq. (108) implies

$$
u_{\mathrm{x}}^{a} R_{a}^{\mathrm{x}}=\gamma_{\mathrm{x}}\left(u^{a}+v_{\mathrm{x}}^{a}\right) R_{a}^{\mathrm{x}}=-\gamma_{\mathrm{x}} \sum_{\mathrm{y} \neq \mathrm{x}} w_{\mathrm{xy}}^{a} \hat{R}_{a}^{\mathrm{xy}}
$$

where

$$
w_{\mathrm{xy}}^{a}=v_{\mathrm{x}}^{a}-v_{\mathrm{y}}^{a} .
$$

A projection of the fluid field equation [cf. Eq. (86)] along the $u_{\mathrm{x}}^{a}$ flow leads to

$$
\Gamma_{\mathrm{x}} u_{\mathrm{x}}^{a} \tilde{\mu}_{a}^{\mathrm{x}} \equiv-\tilde{\mu}^{\mathrm{x}} \Gamma_{\mathrm{x}}=u_{\mathrm{x}}^{a} R_{a}^{\mathrm{x}}
$$

so that

$$
\Gamma_{\mathrm{x}}=\left(\gamma_{\mathrm{x}}^{-1} \tilde{\mu}^{\mathrm{x}}\right)^{-1} \sum_{\mathrm{y} \neq \mathrm{x}} w_{\mathrm{xy}}^{a} \hat{R}_{a}^{\mathrm{xy}}
$$

To further constrain the resistivity, we can use conservation of charge [cf. Eq. (100)], overall charge neutrality, and the Second Law of Thermodynamics $\left(\Gamma_{\mathrm{s}} \geq 0\right)$. The conservation of charge implies

$$
0=\sum_{\mathrm{x}} e^{\mathrm{x}} \Gamma_{\mathrm{x}}=\sum_{\mathrm{x}} \frac{e^{\mathrm{x}}}{\gamma_{\mathrm{x}}^{-1} \tilde{\mu}^{\mathrm{x}}} \sum_{\mathrm{y} \neq \mathrm{x}} w_{\mathrm{xy}}^{a} \hat{R}_{a}^{\mathrm{xy}},
$$

and the Second Law of Thermodynamics takes the form

$$
\Gamma_{\mathrm{s}}=\left(\gamma_{\mathrm{s}}^{-1} \tilde{\mu}^{\mathrm{s}}\right)^{-1} \sum_{\{\mathrm{x} \neq \mathrm{s}\}} w_{\mathrm{sx}}^{a} \hat{R}_{a}^{\mathrm{sx}} \geq 0
$$

We have not yet made any approximation in our system. However, our goal here is to produce a phenomenological model, and so it makes sense to now employ the standard analysis due to Onsager [28] (see also [29, 30]). The point is to introduce a form for the dissipation by identifying thermodynamic fluxes - here the $\hat{R}_{a}^{\mathrm{xy}}$ - and forces — the $w_{\mathrm{xy}}^{a}$. These quantities must be such that they tend to drive the system to equilibrium — the fluids become comoving ( $w_{\mathrm{xy}}^{a}=0$ for all $\mathrm{x}$ and $\mathrm{y}$ ) — while simultaneously maintaining the inequality of Eq. (114). 
Clearly, a model which makes the entropy production manifestly positive-definite will work and so we assume

$$
\hat{R}_{a}^{\mathrm{xy}}=\bar{R}^{\mathrm{xy}} w_{a}^{\mathrm{xy}} \quad \Longrightarrow \quad R_{a}^{\mathrm{xy}}=\bar{R}^{\mathrm{xy}}\left(\delta_{a}^{b}+u_{a} v_{\mathrm{y}}^{b}\right) w_{b}^{\mathrm{xy}}
$$

which leads to

$$
R_{a}^{\mathrm{x}}=\sum_{\mathrm{y} \neq \mathrm{x}}\left[\left(\bar{R}^{\mathrm{yx}} v_{\mathrm{x}}^{b}+\bar{R}^{\mathrm{xy}} v_{\mathrm{y}}^{b}\right) u_{a}+\left(\bar{R}^{\mathrm{yx}}+\bar{R}^{\mathrm{xy}}\right) \delta_{a}^{b}\right] w_{b}^{\mathrm{yx}}
$$

Now introduce

$$
\mathcal{R}^{\mathrm{xy}}=\bar{R}^{\mathrm{yx}}+\bar{R}^{\mathrm{xy}}
$$

(obviously symmetric in $\mathrm{x}$ and $\mathrm{y}$ ) to get

$$
R_{a}^{\mathrm{x}}=\left(\sum_{\mathrm{y} \neq \mathrm{x}} \bar{R}^{\mathrm{xy}} w_{\mathrm{xy}}^{b} w_{b}^{\mathrm{xy}}\right) u_{a}+\sum_{\mathrm{y} \neq \mathrm{x}} \mathcal{R}^{\mathrm{xy}}\left(\delta_{a}^{b}+v_{\mathrm{x}}^{b} u_{a}\right) w_{b}^{\mathrm{yx}} .
$$

Noting that

$$
\Gamma_{\mathrm{x}}=\left(\gamma_{\mathrm{x}}^{-1} \tilde{\mu}^{\mathrm{x}}\right)^{-1} \sum_{\mathrm{y} \neq \mathrm{x}} \bar{R}^{\mathrm{xy}} w_{\mathrm{xy}}^{b} w_{b}^{\mathrm{xy}}
$$

we finally arrive at

$$
R_{a}^{\mathrm{x}}=\left(\gamma_{\mathrm{x}}^{-1} \tilde{\mu}^{\mathrm{x}} \Gamma_{\mathrm{x}}\right) u_{a}+\sum_{\mathrm{y} \neq \mathrm{x}} \mathcal{R}^{\mathrm{xy}}\left(\delta_{a}^{b}+v_{\mathrm{x}}^{b} u_{a}\right) w_{b}^{\mathrm{yx}}
$$

If there are no reactions $\left(\Gamma_{\mathrm{x}}=0\right)$ then

$$
R_{a}^{\mathrm{x}}=\sum_{\mathrm{y} \neq \mathrm{x}} \mathcal{R}^{\mathrm{xy}}\left(\delta_{a}^{b}+v_{\mathrm{x}}^{b} u_{a}\right) w_{b}^{\mathrm{yx}}
$$

Given that the resistivities can depend, in principle, on all of the fluids in the system, any restriction like zero particle creation for a subset of the fluids will have an impact on all the particle creation rates; in particular, the entropy creation rate.

\section{WHAT IS THE MINIMAL MODEL THAT INCLUDES RESISTIVITY?}

Even with this more specific model, there are still a number of degrees of freedom - the undetermined coefficients $\bar{R}^{\mathrm{xy}}$; namely, if we have $N_{c}$ constituents, then for each choice of x, there will be $N_{c}-1$ choices for y, and thus a maximum of $N_{c}\left(N_{c}-1\right)$ coefficients. Note that the condition expressed in Eq. (80) is satisfied identically and so it does not reduce

the number of free $\bar{R}^{\mathrm{xy}}$. The conservation of charge is another matter. Ideally, it is also 
an identity, meaning that the total action $S_{F M C}$ must be constructed in such a way that it incorporates the electromagnetic gauge symmetry. However, in our phenomenological model, we have chosen a form for the $R_{a}^{\mathrm{xy}}$ — it has not been derived as in Eq. (79) and so we must impose charge conservation "by hand", meaning that Eq. (100) is in fact an additional constraint on the system. Hence, a complete specification of the model will require knowing $N_{c}\left(N_{c}-1\right)-1$ of the $\bar{R}^{\text {xy }}$ coefficients.

We will first consider the simplest problem of a two-fluid, two-constituent system where the two types of particles (with labels e and $\mathrm{p}$ ) have equal but opposite charges $\left(-e_{\mathrm{e}}=e_{\mathrm{p}} \equiv\right.$ e). The particle creation rates are

$$
\begin{aligned}
& \gamma_{\mathrm{e}}^{-1} \tilde{\mu}^{\mathrm{e}} \Gamma_{\mathrm{e}}=\bar{R}^{\mathrm{ep}} w_{\mathrm{ep}}^{2} \\
& \gamma_{\mathrm{p}}^{-1} \tilde{\mu}^{\mathrm{p}} \Gamma_{\mathrm{p}}=\bar{R}^{\mathrm{pe}} w_{\mathrm{pe}}^{2}
\end{aligned}
$$

(noting that $w_{\mathrm{ep}}^{a}$ is spatial). Note that charge conservation $\left[c f\right.$. Eq. (113)] implies $\Gamma_{\mathrm{e}}=\Gamma_{\mathrm{p}}$, or

$$
\gamma_{\mathrm{p}}^{-1} \tilde{\mu}^{\mathrm{p}} \bar{R}^{\mathrm{ep}}=\gamma_{\mathrm{e}}^{-1} \tilde{\mu}^{\mathrm{e}} \bar{R}^{\mathrm{pe}}
$$

As the sum of $R_{a}^{\mathrm{e}}$ and $R_{a}^{\mathrm{p}}$ vanishes identically, we see, as expected, that there is only one free component $\bar{R}^{\mathrm{ep}}$. Finally, the two resistivities are

$$
\begin{aligned}
& R_{a}^{\mathrm{e}}=\bar{R}^{\mathrm{ep}}\left[w_{\mathrm{ep}}^{2} u_{a}-\left(1+\frac{\gamma_{\mathrm{p}}^{-1} \tilde{\mu}^{\mathrm{p}}}{\gamma_{\mathrm{e}}^{-1} \tilde{\mu}^{\mathrm{e}}}\right) w_{b}^{\mathrm{ep}}\left(\delta_{a}^{b}+v_{\mathrm{e}}^{b} u_{a}\right)\right] \\
& R_{a}^{\mathrm{p}}=\frac{\gamma_{\mathrm{p}}^{-1} \tilde{\mu}^{\mathrm{p}}}{\gamma_{\mathrm{e}}^{-1} \tilde{\mu}^{\mathrm{e}}} \bar{R}^{\mathrm{ep}}\left[w_{\mathrm{ep}}^{2} u_{a}+\left(1+\frac{\gamma_{\mathrm{e}}^{-1} \tilde{\mu}^{\mathrm{e}}}{\gamma_{\mathrm{p}}^{-1} \tilde{\mu}^{\mathrm{p}}}\right) w_{b}^{\mathrm{ep}}\left(\delta_{a}^{b}+v_{\mathrm{p}}^{b} u_{a}\right)\right] .
\end{aligned}
$$

However, many applications in plasma physics have zero particle creation rates, and we see in this case that the resistivities vanish. Essentially, we are proving that there can be no resistivity without also taking into account heat; i.e. a non-zero entropy creation rate.

The simplest, non-trivial system has the two charged fluids and entropy. The creation rates expand to

$$
\begin{aligned}
& \gamma_{\mathrm{e}}^{-1} \tilde{\mu}^{\mathrm{e}} \Gamma_{\mathrm{e}}=\bar{R}^{\mathrm{ep}} w_{\mathrm{ep}}^{2}+\bar{R}^{\mathrm{es}} w_{\mathrm{es}}^{2}, \\
& \gamma_{\mathrm{p}}^{-1} \tilde{\mu}^{\mathrm{p}} \Gamma_{\mathrm{p}}=\bar{R}^{\mathrm{pe}} w_{\mathrm{pe}}^{2}+\bar{R}^{\mathrm{ps}} w_{\mathrm{ps}}^{2}, \\
& \gamma_{\mathrm{s}}^{-1} \tilde{\mu}^{\mathrm{s}} \Gamma_{\mathrm{s}}=\bar{R}^{\mathrm{se}} w_{\mathrm{se}}^{2}+\bar{R}^{\mathrm{sp}} w_{\mathrm{sp}}^{2},
\end{aligned}
$$


and the resistivities are

$$
\begin{aligned}
& R_{a}^{\mathrm{e}}=\left(\bar{R}^{\mathrm{ep}} w_{\mathrm{pe}}^{2}+\bar{R}^{\mathrm{es}} w_{\mathrm{se}}^{2}\right) u_{a}+\left(\mathcal{R}^{\mathrm{pe}} w_{b}^{\mathrm{pe}}+\mathcal{R}^{\mathrm{se}} w_{b}^{\mathrm{se}}\right)\left(\delta_{a}^{b}+v_{\mathrm{e}}^{b} u_{a}\right) \\
& R_{a}^{\mathrm{p}}=\left(\bar{R}^{\mathrm{pe}} w_{\mathrm{ep}}^{2}+\bar{R}^{\mathrm{ps}} w_{\mathrm{sp}}^{2}\right) u_{a}+\left(\mathcal{R}^{\mathrm{ep}} w_{b}^{\mathrm{ep}}+\mathcal{R}^{\mathrm{sp}} w_{b}^{\mathrm{sp}}\right)\left(\delta_{a}^{b}+v_{\mathrm{p}}^{b} u_{a}\right) \\
& R_{a}^{\mathrm{s}}=\left(\bar{R}^{\mathrm{se}} w_{\mathrm{es}}^{2}+\bar{R}^{\mathrm{sp}} w_{\mathrm{ps}}^{2}\right) u_{a}+\left(\mathcal{R}^{\mathrm{es}} w_{b}^{\mathrm{es}}+\mathcal{R}^{\mathrm{ps}} w_{b}^{\mathrm{ps}}\right)\left(\delta_{a}^{b}+v_{\mathrm{s}}^{b} u_{a}\right)
\end{aligned}
$$

Charge conservation gives

$$
0=\left(\frac{1}{\gamma_{\mathrm{p}}^{-1} \tilde{\mu}^{\mathrm{p}}} \bar{R}^{\mathrm{pe}}-\frac{1}{\gamma_{\mathrm{e}}^{-1} \tilde{\mu}^{\mathrm{e}}} \bar{R}^{\mathrm{ep}}\right) w_{\mathrm{pe}}^{2}+\left(\frac{1}{\gamma_{\mathrm{p}}^{-1} \tilde{\mu}^{\mathrm{p}}} \bar{R}^{\mathrm{ps}} w_{\mathrm{ps}}^{2}-\frac{1}{\gamma_{\mathrm{e}}^{-1} \tilde{\mu}^{\mathrm{e}}} \bar{R}^{\mathrm{es}} w_{\mathrm{es}}^{2}\right)
$$

and the Second Law $[c f$. Eq. (114)] implies

$$
\bar{R}^{\text {se }}, \bar{R}^{\text {sp }} \geq 0
$$

If we now assume that there is no charge creation, then

$$
\begin{aligned}
& 0=\bar{R}^{\mathrm{ep}} w_{\mathrm{ep}}^{2}+\bar{R}^{\mathrm{es}} w_{\mathrm{es}}^{2}, \\
& 0=\bar{R}^{\mathrm{pe}} w_{\mathrm{ep}}^{2}+\bar{R}^{\mathrm{ps}} w_{\mathrm{ps}}^{2} .
\end{aligned}
$$

Unlike before, we can satisfy these conditions with something as simple as requiring the coefficients $\bar{R}^{\text {ep }}, \bar{R}^{\text {pe }}, \bar{R}^{\text {es }}$, and $\bar{R}^{\text {ps }}$ to vanish. ${ }^{2}$ This will leave us with only two free coefficients, $\bar{R}^{\text {se }}$ and $\bar{R}^{\text {sp }}$, and resistivities of the form

$$
\begin{aligned}
& R_{a}^{\mathrm{e}}=\bar{R}^{\mathrm{se}} w_{b}^{\mathrm{se}}\left(\delta_{a}^{b}+v_{\mathrm{e}}^{b} u_{a}\right), \\
& R_{a}^{\mathrm{p}}=\bar{R}^{\mathrm{sp}} w_{b}^{\mathrm{sp}}\left(\delta_{a}^{b}+v_{\mathrm{p}}^{b} u_{a}\right) \\
& R_{a}^{\mathrm{s}}=\left(\bar{R}^{\mathrm{se}} w_{\mathrm{es}}^{2}+\bar{R}^{\mathrm{sp}} w_{\mathrm{ps}}^{2}\right) u_{a}+\left(\bar{R}^{\mathrm{se}} w_{b}^{\mathrm{es}}+\bar{R}^{\mathrm{sp}} w_{b}^{\mathrm{ps}}\right)\left(\delta_{a}^{b}+v_{\mathrm{s}}^{b} u_{a}\right) .
\end{aligned}
$$

Perhaps the most important point of developing this kind of phenomenological model is to show that, even without specific forms for the $n_{A B C}^{\mathrm{x}}$ and $\Lambda$, the multi-fluid formalism is robust enough to build increasingly complex models without first having to perform microphysical calculations. Of course, we would still need some insight from microphysics; e.g. to determine $\bar{R}^{\text {se }}$ and $\bar{R}^{\text {sp }}$.

\footnotetext{
${ }^{2}$ Since $w_{\mathrm{ep}}^{2}, w_{\mathrm{es}}^{2}$, and $w_{\mathrm{ps}}^{2}$ are linearly independent, this is tantamount to assuming that the $\bar{R}^{\mathrm{xy}}$ have negligible dependence on the relative velocities.
} 


\section{CONCLUSIONS AND FOLLOW-ON WORK}

The relativistic fluid system is the backbone of modeling many astrophysical phenomena, cosmology and high-energy physics. Here, we have taken a unique step in the development of the fluid modeling scheme: an action principle has been used to build a system of field equations for relativistic plasmas including resistivity. This is a "first principles" approach which is logically concise in the sense that many of the assumptions about the system's physics can be traced to the initial phase of constructing the action; in particular, it was straightforward to take the action principle for dissipative, relativistic fluids from [16] and add to it the standard action for electromagnetic fields and the usual Coulomb coupling of the charged fluxes to the electromagnetic four-potential.

The present discussion is complemented by two companion papers. In [17] we use this work's results to develop a fully relativistic framework that allows for four (fluid) components: normal and superconducting currents, heat flow, and a final component with normal and superfluid flows. The purpose of the model is to extend and make contact with (in the appropriate limit) ideal magnetohydrodynamics. A key component of the framework is the insertion of a suitable family of observers of the fluid flow, who basically provide a fibration of spacetime. While the model is suitable to describe isolated superfluid neutron stars, it is not appropriate for numerical simulations of (say) merging neutron stars. Progress in this direction is made in [18], which connects with the present discussion through use of a $3+1$ foliation of spacetime.

While our focus here was on the resistivity, there is a clear process for building on these results by adding in other dissipation channels (such as those arising from bulk and shear viscosities) already included in the action principle of Andersson and Comer [16]. Basically, we may follow the procedure presented here, with the only change being to include terms like the matter space metrics $g_{\mathrm{x}}^{A B}$ and $g_{\mathrm{xy}}^{A B}\left[c f\right.$. Eqs. (18) and (20)] in the variation of $n_{A B C}^{\mathrm{x}}$ [cf. Eq. (72)]. This speaks to the power of having a first principles approach to developing the overall form of the field equations, even if details of the formalism still will require

microphysics for dissipation coefficients [such as $\bar{R}^{\text {se }}$ and $\bar{R}^{\text {sp }}$ in the phenomenological model; cf. Eq. (139)].

To conclude, the variational approach has allowed us to make significant progress, both formal and practical, on a problem which is central to modern relativistic astrophysics. The 
framework we have developed is ready to be applied and we expect to report progress on a set of relevant problems in the near future.

\section{Acknowledgments}

NA and IH gratefully acknowledge support from the STFC.

\section{Appendix A: Vector Potential "Entrainment"}

It may be worth noting that the Coulomb action in Eq. (62) depends on the metric, and the combination $n_{\mathrm{x}}^{a} A_{a}$, which is exactly of the entrainment form, if we consider it as part of the fluid action $S_{M}$ (cf. Eq. (56)). This means that, at least formally, we can consider $\Lambda$ to be a functional of the set $\left\{n_{\mathrm{x}}^{2}, n_{\mathrm{xy}}^{2}, n_{\mathrm{x}}^{a} A_{a}\right\}$. This may be interesting as there are general constraints that can be had for plasmas if we make statements about gauge-invariance of the total fluid/plasma action and the vector potential entrainment. It is plausible that more general forms for the entrainment could lead to known results in, say, non-linear media in a more efficient way.

It is straightforward to work through the steps of varying the new action and obtaining the equations of motion:

$$
S=S_{M}\left(n_{\mathrm{x}}^{2}, n_{\mathrm{xy}}^{2}, A_{\mathrm{x}}^{2}\right)+S_{M a x}\left(A_{a}\right)
$$

where

$$
A_{\mathrm{x}}^{2} \equiv-n_{\mathrm{x}}^{a} A_{a}
$$

Next,

$$
\begin{aligned}
\delta S= & \int_{\mathcal{M}} \mathrm{d}^{4} x \sqrt{-g}\left[\sum_{\mathrm{x}} \mu_{a}^{\mathrm{x}} \delta n_{\mathrm{x}}^{a}-\sum_{\mathrm{x}} \frac{\partial \Lambda}{\partial A_{\mathrm{x}}^{2}}\left(A_{a} \delta n_{\mathrm{x}}^{a}+n_{\mathrm{x}}^{a} \delta A_{a}\right)\right. \\
& \left.+\frac{1}{2}\left(\Lambda g^{a b}+\sum_{\mathrm{x}} n_{\mathrm{x}}^{a} \mu_{\mathrm{x}}^{b}\right) \delta g_{a b}\right]+\frac{1}{4 \pi} \int_{\mathcal{M}} \mathrm{d}^{4} x \sqrt{-g}\left(\nabla_{a} F^{a b}\right) \delta A_{b} \\
& -\frac{1}{32 \pi} \int_{\mathcal{M}} \mathrm{d}^{4} x \sqrt{-g}\left(F_{c d} F^{c d} g^{a b}-4 F^{a c} F^{b}{ }_{c}\right) \delta g_{a b} \\
= & \int_{\mathcal{M}} \mathrm{d}^{4} x \sqrt{-g}\left\{-\sum_{\mathrm{x}}\left(f_{a}^{\mathrm{x}}+\Gamma_{\mathrm{x}} \tilde{\mu}_{a}^{\mathrm{x}}-R_{a}^{\mathrm{x}}\right) \xi_{\mathrm{x}}^{a}-\frac{1}{4 \pi}\left(\nabla_{b} F^{a b}-4 \pi \sum_{\mathrm{x}} \mathcal{Q}_{\mathrm{x}} n_{\mathrm{x}}^{a}\right) \delta A_{a}\right. \\
& \left.+\frac{1}{2}\left[\Psi g^{a b}+\sum_{\mathrm{x}} n_{\mathrm{x}}^{a} \mu_{\mathrm{x}}^{b}-\frac{1}{16 \pi}\left(F_{c d} F^{c d} g^{a b}-4 F^{a c} F^{b}{ }_{c}\right)\right] \delta g_{a b}\right\}
\end{aligned}
$$


where

$$
\tilde{\mu}_{a}^{\mathrm{x}}=\mu_{a}^{\mathrm{x}}+\mathcal{Q}_{\mathrm{x}} A_{a} \quad, \quad \mathcal{Q}_{\mathrm{x}}\left(n_{\mathrm{x}}^{2}, n_{\mathrm{xy}}^{2}, A_{\mathrm{x}}^{2}\right) \equiv-\frac{\partial \Lambda}{\partial A_{\mathrm{x}}^{2}} .
$$

We recover the minimal coupling when

$$
\mathcal{Q}_{\mathrm{x}}=e_{\mathrm{x}}
$$

What happens if we now impose gauge-invariance on the whole system? We consider a variation of only the vector potential which is of the form

$$
\delta A_{a}=\nabla_{a} \delta \phi
$$

Taking into account the identity in Eq. (100), we see that

$$
\sum_{\mathrm{x}} \nabla_{a}\left(\mathcal{Q}_{\mathrm{x}} n_{\mathrm{x}}^{a}\right)=0
$$

This can also be written as

$$
\sum_{\mathrm{x}}\left(\mathcal{Q}_{\mathrm{x}} \Gamma_{\mathrm{x}}+n_{\mathrm{x}} \frac{\mathrm{d} \mathcal{Q}_{\mathrm{x}}}{\mathrm{d} \tau_{\mathrm{x}}}\right)=0
$$

If $\mathcal{Q}_{\mathrm{x}}$ depends on only $X_{\mathrm{x}}^{A}$ (it is Lie-dragged by $u_{\mathrm{x}}^{a}$ ) then this reduces to

$$
\sum_{\mathrm{x}} \mathcal{Q}_{\mathrm{x}} \Gamma_{\mathrm{x}}=0
$$

[1] N. Andersson and G. L. Comer, Living Reviews in Relativity 10, 1 (2007).

[2] B. Müller, The Status of Multi-Dimensional Core-Collapse Supernova Models, preprint (2016), ArXiv:1608.03274.

[3] H. Andresen, Haakon, B. Mueller, E. Mueller and H.-T. Janka, Gravitational Wave Signals from 3D Neutrino Hydrodynamics Simulations of Core-Collapse Supernovae preprint (2016), ArXiv:1607.05199

[4] L. Baiotti and L. Rezzolla, Binary neutron-star mergers: a review of Einstein's richest laboratory, preprint (2016), ArXiv:1607.03540.

[5] K. Dionysopoulou, D. Alic, and L. Rezzolla, Phys. Rev. D 92084064 (2015).

[6] W. Israel and J. M. Stewart, Ann. Phys. 118, 341 (1979). 
[7] W. Israel and J. M. Stewart, Royal Society of London Proceedings Series A 365, 43 (1979).

[8] M. Rangamani, Class. Quantum Grav. 26, 224003 (2009).

[9] C. S. Lopez-Monsalvo and N. Andersson, Proceedings of the Royal Society of London Series A 467, 738 (2011).

[10] N. Andersson and C. S. Lopez-Monsalvo, Classical and Quantum Gravity 28, 195023 (2011).

[11] N. Andersson, Phys. Rev. D 86, 043002 (2012).

[12] A. H. Taub, Phys. Rev. 94, 1468 (1954).

[13] B. Carter, in Relativistic Fluid Dynamics (Noto, 1987), edited by A. Anile and M. ChoquetBruhat (Springer-Verlag, Heidelberg, Germany, 1989), vol. 1385 of Lecture Notes in Mathematics, pp. 1-64.

[14] G. L. Comer and D. Langlois, Class. Quant. Grav. 10, 2317 (1993).

[15] G. L. Comer and D. Langlois, Class. Quant. Grav. 11, 709 (1994).

[16] N. Andersson and G. L. Comer, Class. Quant. Grav. 32, 075008 (2015), 1306.3345.

[17] N. Andersson, K. Dionysopoulou, I. Hawke, and G. L. Comer, in preparation (2016).

[18] N. Andersson, I. Hawke, K. Dionysopoulou, and G. L. Comer, in preparation (2016).

[19] C. W. Misner, K. S. Thorne, and J. A. Wheeler, Gravitation (San Francisco, US: W.H. Freeman and Co., 1973).

[20] G. Strang, Linear Algebra and Its Applications (New York: Academic Press, 1980), 2nd ed.

[21] R. M. Wald, General Relativity (Chicago, US: University of Chicago Press, 1984).

[22] L. E. Reichl, A Modern Course in Statistical Physics, 2nd Edition (Wiley-VCH, 1998).

[23] A. F. Andreev and E. P. Bashkin, Sov. Phys. JETP 42, 164 (1975).

[24] M. Borumand, R. Joynt, and W. Kluzniak, Phys. Rev. C 54, 2745 (1996).

[25] G. L. Comer and R. Joynt, Phys. Rev. D 68, 023002 (2003).

[26] N. Chamel and P. Haensel, Phys. Rev. C 73, 045802 (2006), arXiv:nucl-th/0603018.

[27] N. Andersson and G. L. Comer, Proceedings of the Royal Society A: Mathematical, Physical and Engineering Science 466, 1373 (2010).

[28] L. Onsager, Phys. Rev. 37, 405 (1931).

[29] N. Andersson and G. L. Comer, Class. Quant. Grav. 23, 5505 (2006).

[30] B. Haskell, N. Andersson and G.L. Comer, Phys. Rev. D 86, 063002 (2012). 